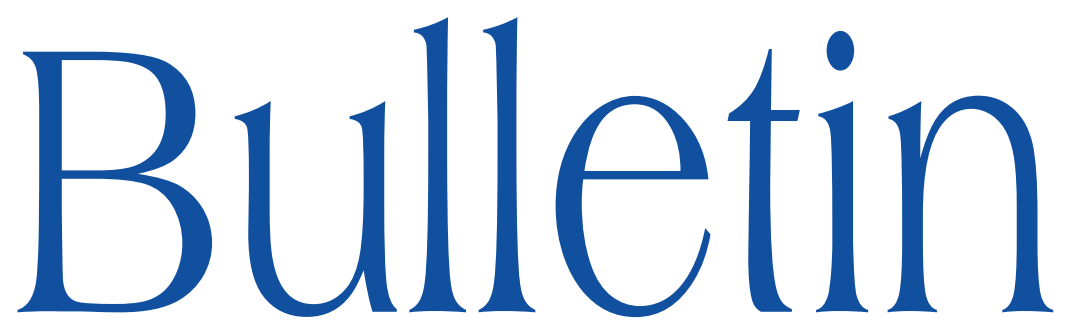

de la SOCIÉTÉ MATHÉMATIQUE DE FRANCE

\title{
UN THÉORÈME DE BEILINSON-BERNSTEIN POUR LES D-MODULES ARITHMÉTIQUES
}

Christine Noot-Huyghe

Tome 137

Fascicule 2

2000 


\title{
UN THÉORÈME DE BEILINSON-BERNSTEIN POUR LES $\mathcal{D}$-MODULES ARITHMÉTIQUES
}

\author{
PAR Christine Noot-Huyghe
}

\begin{abstract}
Résumé. - Un résultat important de la théorie des groupes, démontré indépendemment dans les années 80 par Beilinson et Bernstein, Brylinski et Kashiwara, est un résultat d'affinité des $\mathcal{D}$-modules sur la variété de drapeaux d'un groupe réductif sur le corps des nombres complexes. Nous donnons ici un analogue arithmétique de ce résultat, pour la catégorie des $\mathcal{D}$-modules arithmétiques sur la variété de drapeaux d'un groupe réductif sur un anneau de valuation discrète complet d'inégales caractéristiques $(0, p)$.
\end{abstract}

Abstract ( $A$ Beilinson-Bernstein theorem for arithmetic $\mathcal{D}$-modules)

An important result of group theory, independently proved during the years ' 80 , by Beilinson and Bernstein, Brylinski and Kashiwara, is an affinity result for $\mathcal{D}$-modules on the flag variety of a reductive group over the field of complex numbers. We give here an arithmetic analogue of this result, for the category of arithmetic $\mathcal{D}$-modules on the flag variety of a reductive group over a discrete valuation ring of inequal characteristics $(0, p)$.

Texte reçu le 7 mai 2007, révisé le 3 juillet 2008, accepté le 12 septembre 2008

Christine Noot-Huyghe, IRMA, Université Louis Pasteur, 7, rue René Descartes, 67084 Strasbourg Cedex, France - E-mail : huyghe@math.u-strasbg.fr Url : http://www-irma.u-strasbg.fr/ huyghe

Classification mathématique par sujets (2000). — 14F17, 14F30.

Mots clefs. - Localisation, $\mathcal{D}$-modules arithmétiques, variétés de drapeaux, théorèmes d'acyclicité.

This work has been supported by the research network Arithmetic Algebraic Geometry of the European Community (Programme FP6, contrat MRTN-CT2003-504917). 


\section{Introduction}

Soit $V$ un anneau de valuation discrète, d'inégales caractéristiques $(0, p)$. On considère ici les deux situations suivantes :

1. $S=\operatorname{spec} V$, le spectre de $V, X$ est un $S$-schéma noethérien,

2. $\mathcal{S}=\operatorname{Spf} V$, le spectre formel de $V, \mathcal{X}$ un schéma formel noethérien sur $\mathcal{S}$.

Soit $\mathcal{A}$ un faisceau cohérent de $\mathcal{O}_{X}$-algèbres, quasi-cohérent comme $\mathcal{O}_{X}$-module

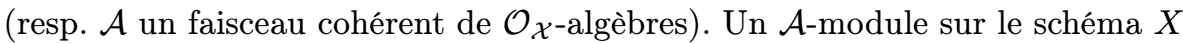
sera dit quasi-cohérent s'il est un $\mathcal{O}_{X}$-module quasi-cohérent. On dit que $X$ (resp. $\mathcal{X}$ ) est $\mathcal{A}$-affine si les deux propriétés suivantes sont vérifiées :

(i) Pour tout $\mathcal{A}$-module quasi-cohérent $\mathcal{M}$ sur $X$ (resp. tout $\mathcal{A}$-module cohérent $\operatorname{sur} \mathcal{X}$ ) et tout $n \geq 1$ on a les égalités $H^{n}(X, \mathcal{M})=0$ (resp. $\left.H^{n}(\mathcal{X}, \mathcal{M})=0\right)$.

(ii) Le foncteur $\Gamma$ établit une équivalence de catégories entre la catégorie des $\mathcal{A}$-modules quasi-cohérents (resp. des $\mathcal{A}$-modules cohérents) et la catégorie $\operatorname{des} \Gamma(X, \mathcal{A})$-modules (resp. des $\Gamma(\mathcal{X}, \mathcal{A})$-modules de type fini).

Un énoncé important de la théorie des groupes est le théorème de BeilinsonBernstein : soit $G$ un groupe semi-simple sur $\mathbf{C}, X$ une variété de drapeaux de $G, \mathcal{D}_{X}$ le faisceau des opérateurs différentiels sur $\mathcal{X}$, alors $X$ est $\mathcal{D}_{X}$-affine. On se propose de donner ici un analogue arithmétique de cet énoncé, dans la situation qui suit. Soit $G$ un groupe semi-simple sur $S, \rho$ la demi-somme des racines positives de $G, P$ un sous-groupe parabolique de $G, X=G / P, \mathcal{X}$ le schéma formel obtenu en complétant $X$ le long de la fibre spéciale de $S$. Ce schéma est lisse et on peut s'intéresser au faisceau des opérateurs différentiels arithmétiques sur $\mathcal{X}$ construit par Berthelot, que nous noterons $\mathcal{D}_{\mathcal{X}, \mathbf{Q}}^{\dagger}$. On

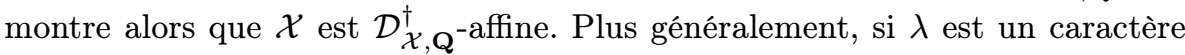
de $G, \mathcal{L}(\lambda)$ le faisceau inversible sur $\mathcal{X}$ associé au caractère $\lambda$ (cf A.4), et $\mathcal{D}_{\mathcal{X}, \mathbf{Q}}^{\dagger}(\lambda)$ le faisceau des opérateurs différentiels arithmétiques à valeurs dans $\mathcal{L}(\lambda)$, alors $\mathcal{X}$ est $\mathcal{D}_{\mathcal{X}, \mathbf{Q}}^{\dagger}(\lambda)$-affine pour tout poids $\lambda$ tel que $\lambda+\rho$ est dominant et régulier.

En caractéristique 0 , pour le faisceau $\mathcal{D}(\lambda)$ tel que $\lambda+\rho$ est dominant et régulier, le résultat est démontré indépendamment par Beilinson-Bernstein ([2]) et par Brylinski-Kashiwara ([8]) et joue un rôle essentiel dans la démonstration de la conjecture de multiplicité de Kazhdan-Lusztig ([18]).

En caractéristique $p>0$, Haastert a montré que cet énoncé d'affinité était vérifié pour les espaces projectifs, ainsi que pour la variété de drapeaux de $S L_{3}$ ([13]). En revanche, Kashiwara-Lauritzen ont donné un contre-exemple à cet énoncé, pour le faisceau usuel $\mathcal{D}$ ([17]) défini dans [12] et pour la grassmanienne des sous-espaces vectoriels de dimension 2 d'un espace de dimension 5. Enfin,

TOME $137-2009-\mathrm{N}^{\mathrm{O}} 2$ 
Bezrukavnikov, Mirkovic, Rumynin ont montré (3.2 de [5]) un analogue de ce résultat d'affinité en passant à la catégorie dérivée bornée des $\mathcal{D}^{(0)}$-modules cohérents sur $X$ (i.e. les opérateurs différentiels sans puissances divisées) et sous la condition que $p$ soit strictement plus grand que le nombre de Coxeter de $G$.

En caractéristique mixte, le résultat a été montré pour les espaces projectifs ([14]). Dans ce cas, on utilise de façon cruciale le fait que le faisceau tangent est très ample, ce qui caractérise l'espace projectif. Le point clé pour les variétés de drapeaux est que la catégorie des $\mathcal{D}_{\mathcal{X}, \mathbf{Q}}^{\dagger}$-modules cohérents est engendrée par les modules induits (i.e. du type $\mathcal{D}_{\mathcal{X}, \mathbf{Q}}^{\dagger} \otimes_{\mathcal{O}_{\mathcal{X}}} \mathcal{E}$ où $\mathcal{E}$ est un $\mathcal{O}_{\mathcal{X}}$-module cohérent). On utilise cette propriété pour montrer que si le résultat de $\mathcal{D}$-affinité est vrai algébriquement, pour le faisceau $\mathcal{D}_{X_{K}}$, alors il est vrai pour le faisceau $\mathcal{D}_{\mathcal{X}, \mathbf{Q}}^{\dagger}$ sur le schéma formel $\mathcal{X}$ (théorème 2.1).

Nous n'aborderons pas ici l'aspect localisation de Lie $(G)$-modules (ou plutôt des modules sur la complétion faible de $\operatorname{Lie}(G))$, qui est bien entendu sousjacent et fera l'objet d'un article ultérieur.

Nous remercions le referee pour sa lecture attentive et ses suggestions pertinentes pour améliorer la rédaction de ce texte.

\section{Notations-Rappels}

1.1. Notations. - Dans toute la suite, on note $K$ le corps des fractions de $V$, $\pi$ une uniformisante et $k$ le corps résiduel de $V$. Soit $G$ un groupe semi-simple sur $S, P$ un sous-groupe parabolique de $G$. On rappelle que, par définition ([9]), un sous-groupe parabolique est plat sur $S$. Comme $S$ est le spectre d'un anneau de Dedekind, le quotient $X=G / P$ est un $S$-schéma ([1]). On note $N$ la dimension de $X$, et $\mathcal{X}$ le schéma formel associé par complétion à $X$.

D'une façon générale, si $Z$ est un $S$-schéma, la lettre cursive $\mathcal{Z}$ désignera le schéma formel obtenu en complétant $Z$ le long de l'idéal $\pi, Z_{k}$ la fibre spéciale $Z_{k}=\operatorname{spec} k \times_{S} Z$ et $Z_{K}$ la fibre générique $Z_{K}=\operatorname{spec} K \times_{S} Z$. On posera $i_{Z}$ l'immersion fermée $Z_{k} \hookrightarrow Z$ et $j_{Z}$ l'immersion ouverte $Z_{K} \rightarrow Z$ (on omettra éventuellement le $Z$ dans les notations quand le contexte sera clair). On notera aussi

$$
Z_{i}=Z \times_{S} \operatorname{spec}\left(S / \pi^{i+1} S\right) .
$$

Pour un faisceau $\mathcal{E}$ sur un $S$-schéma $Z$, on notera $\mathcal{E}_{k}=i^{*} \mathcal{E}$ et $\mathcal{E}_{K}=j^{*} \mathcal{E}$. 
1.2. Coefficients $p$-adiques. - Fixons un entier $m$. Si $k_{i} \in \mathbf{N}$, on introduit $q_{k_{i}}$ le quotient de la division euclidienne de $k_{i}$ par $p^{m}$ et pour un multi-indice $\underline{k}=\left(k_{1}, \ldots, k_{N}\right)$ on définit

$$
q_{\underline{k}} !=\prod_{i=1}^{N} q_{k_{i}} !
$$

Pour $k \leq l \in \mathbf{N}$, on pose

$$
\begin{gathered}
\left\{\begin{array}{l}
l \\
k
\end{array}\right\}=\frac{q_{k} !}{q_{k} ! q_{l-k} !}, \\
\left\langle\begin{array}{l}
l \\
k
\end{array}\right\rangle=\left(\begin{array}{l}
l \\
k
\end{array}\right)\left\{\begin{array}{l}
l \\
k
\end{array}\right\}^{-1} \in \mathbf{Z}_{(p)},
\end{gathered}
$$

et pour des multi-indices $\underline{k}, \underline{l} \in \mathbf{N}^{N}$, tels que $\underline{k} \leq \underline{l}$ (i.e. $k_{i} \leq l_{i}$ pour tout $1 \leq i \leq N)$

$$
\left\langle\begin{array}{l}
\underline{l} \\
\underline{k}
\end{array}\right\rangle=\prod_{i=1}^{N}\left\langle\begin{array}{c}
l_{i} \\
k_{i}
\end{array}\right\rangle .
$$

On définit de façon analogue les coefficients $\left\{\frac{l}{\underline{k}}\right\}$ et $\left(\frac{l}{\underline{k}}\right)$.

Décrivons maintenant les différents faisceaux d'opérateurs différentiels intervenant dans cette situation.

1.3. Opérateurs différentiels arithmétiques. - Dans cette partie, $X$ est un $S$ schéma lisse et $\mathcal{X}$ est son complété formel le long de l'idéal engendré par $\pi$. On décrit les différents faisceaux d'opérateurs différentiels en coordonnées locales. Nous renvoyons à [12] et à [3] pour une définition intrinsèque de ces faisceaux. Soit $U$ un ouvert affine lisse de $X, x_{1}, \ldots, x_{N}$ une famille de coordonnées locales sur $X, d x_{1}, \ldots, d x_{N}$ une base de $\Omega_{X}^{1}(U), \partial_{1}, \ldots, \partial_{N}$ la base duale de $\mathcal{T}_{X}(U)$. Si $k_{i} \in \mathbf{N}$, on note $\partial_{i}^{\left[k_{i}\right]}=\partial_{i} / k_{i}$ ! et pour un multi-indice $\underline{\partial}^{[k]}=\prod_{i=1}^{N} \partial_{i}^{\left[k_{i}\right]}$. Alors on a la description suivante ([12])

$$
\mathcal{D}_{X}(U)=\left\{\sum_{\text {finies }} a_{\underline{k}} \underline{\partial}^{[\underline{k}]} \mid a_{\underline{k}} \in \mathcal{O}_{X}(U)\right\} .
$$

Donnons maintenant une description des faisceaux d'opérateurs différentiels construits par P. Berthelot.

Soit $m \in \mathbf{N}$. P. Berthelot introduit les faisceaux $\mathcal{D}_{X}^{(m)}$, ainsi que $\widehat{\mathcal{D}}_{\mathcal{X}}^{(m)}$, leur complété $p$-adique sur $\mathcal{X}$. Notons

$$
\underline{\partial}^{\langle\underline{k}\rangle}(m)=q_{\underline{k}} \underline{\partial}^{[k]} .
$$

TOME $137-2009-\mathrm{N}^{\mathrm{O}} 2$ 
On a alors les descriptions suivantes

$$
\begin{gathered}
\mathcal{D}_{X}^{(0)}(U)=\left\{\sum_{\text {finies }} a_{\underline{k}} \underline{\partial}^{\underline{k}} \mid a_{\underline{k}} \in \mathcal{O}_{X}(U)\right\}, \\
\mathcal{D}_{X}^{(m)}(U)=\left\{\sum_{\text {finies }} a_{\underline{k}} \underline{\partial}^{\langle\underline{k}\rangle_{(m)}} \mid a_{\underline{k}} \in \mathcal{O}_{X}(U)\right\} .
\end{gathered}
$$

Ces faisceaux d'anneaux sont filtrés par les sous-faisceaux de $\mathcal{O}_{X}$-modules $\mathcal{D}_{X, t}^{(m)}$ des opérateurs différentiels d'ordre $\leq t$ pour $t \in \mathbf{N}$, et on a

$$
\mathcal{D}_{X, t}^{(m)}(U)=\left\{\sum_{|\underline{k}| \leq t} a_{\underline{k}} \underline{\partial}^{\langle\underline{k}\rangle_{(m)}} \mid a_{\underline{k}} \in \mathcal{O}_{X}(U)\right\} .
$$

Pour $m=+\infty$, les définitions s'étendent naturellement (tous les coefficients $q_{k}$ ! sont alors égaux à 1 ) et on retrouve le faisceau usuel $\mathcal{D}_{X}$. Les faisceaux $\mathcal{D}_{X}^{(m)}$ forment un système inductif, ainsi que leurs complétés $p$-adiques $\widehat{\mathcal{D}}_{\mathcal{X}}^{(m)}$. On posera

$$
\mathcal{D}_{\mathcal{X}, \mathbf{Q}}^{\dagger}=\underset{m}{\lim } \widehat{\mathcal{D}}_{\mathcal{X}, \mathbf{Q}}^{(m)}
$$

Les faisceaux $\mathcal{D}_{X}^{(m)}$ sont à sections noethériennes sur les ouverts affines, c'est donc aussi le cas des faisceaux $\widehat{\mathcal{D}}_{\mathcal{X}, \mathbf{Q}}^{(m)}$, qui sont cohérents. On en déduit, via un théorème de platitude, que le faisceau $\mathcal{D}_{\mathcal{X}, \mathbf{Q}}^{\dagger}$ est cohérent.

Plus précisément, la structure de l'algèbre graduée de $\mathcal{D}_{X}^{(m)}$ est décrite en 1.3.7.3 de [14] en termes d'algèbre symétrique de niveau $m$ du faisceau tangent.

Rappelons comment est construite l'algèbre symétrique de niveau $m$ d'un $\mathcal{O}_{X}$-module localement libre $\mathcal{E}$ (section 1 de [14]).

1.4. Algèbres symétriques de niveau $m$. - Pour les définitions relatives aux $m$ $\mathrm{PD}$-structures, on se reportera à [3]. Soit $\mathcal{E}$ un $\mathcal{O}_{X}$-module localement libre. Le faisceau d'algèbres symétriques $\mathbf{S}\left(\mathcal{E}^{\vee}\right)$ est gradué et muni de l'idéal d'augmentation $I\left(\mathcal{E}^{\vee}\right)=\bigoplus_{n \geq 1} S_{n}\left(\mathcal{E}^{\vee}\right)$. Par définition, $\boldsymbol{\Gamma}_{(m)}\left(\mathcal{E}^{\vee}\right)$ est la $m$-PD-enveloppe du couple $\left(\mathbf{S}\left(\mathcal{E}^{\vee}\right), I\left(\mathcal{E}^{\vee}\right)\right)$. Ce faisceau d'algèbres est muni d'un $m$-PD-idéal $\overline{\mathcal{I}}$, définissant une $m$-PD-filtration et on définit

$$
\Gamma_{(m)}^{n}\left(\mathcal{E}^{\vee}\right)=\Gamma_{(m)}\left(\mathcal{E}^{\vee}\right) / \overline{\mathcal{I}}^{\{n+1\}}
$$

On pose enfin

$$
\mathbf{S}^{(m)}(\mathcal{E})=\bigcup_{n} \mathcal{H o m}_{\mathcal{O}_{X}}\left(\Gamma_{(m)}^{n}\left(\mathcal{E}^{\vee}\right), \mathcal{O}_{X}\right),
$$

qui est (1.3.3 de [14]) un faisceau de $\mathcal{O}_{X}$-algèbres commutatives graduées par

$$
\mathbf{S}^{(m)}(\mathcal{E})=\bigoplus_{n \in \mathbf{N}} S_{n}^{(m)}(\mathcal{E}), \text { où } \mathrm{S}_{\mathrm{n}}^{(\mathrm{m})}(\mathcal{E})=\mathcal{H}_{\mathrm{om}_{\mathcal{O}_{\mathrm{X}}}}\left(\overline{\mathcal{I}}^{\{\mathrm{n}\}} / \overline{\mathcal{I}}^{\{\mathrm{n}+1\}}, \mathcal{O}_{\mathrm{X}}\right) .
$$


Les modules $S_{n}^{(m)}(\mathcal{E})$ sont localement libres de rang fini et le faisceau $\mathbf{S}^{(m)}(\mathcal{E})$ est un faisceau d'algèbres localement noethériennes. Ces constructions définissent des foncteurs covariants $\boldsymbol{\Gamma}_{(m)}$ et $\mathbf{S}^{(m)}$ de la catégorie des $\mathcal{O}_{X}$-modules localement libres vers la catégorie des faisceaux d'algèbres commutatives graduées.

Si $y_{1}, \ldots y_{N}$ sont une base locale de $\mathcal{E}$ sur un ouvert $U$ de $X$, le faisceau $\mathbf{S}_{n}^{(m)}(\mathcal{E})$ admet pour base sur $U$ des éléments

$$
\underline{y}^{\langle\underline{k}\rangle}=y_{1}^{\left\langle k_{1}\right\rangle} y_{2}^{\left\langle k_{2}\right\rangle} \cdots y_{N}^{\left\langle k_{N}\right\rangle} \text { tels que }|\underline{k}|=n .
$$

De plus ces éléments vérifient

$$
\underline{y}^{\langle\underline{k}\rangle} \cdot \underline{y}^{\langle\underline{l}\rangle}=\left\langle\begin{array}{c}
\underline{k}+\underline{l} \\
\underline{k}
\end{array}\right\rangle \underline{y}^{\langle\underline{k}+\underline{l}\rangle} .
$$

Nous aurons besoin de la propriété de dévissage suivante (1.3.9 de [14]). Soit $0 \rightarrow \mathcal{E} \rightarrow \mathcal{F} \rightarrow \mathcal{G} \rightarrow 0$ une suite exacte de $\mathcal{O}_{X}$-modules localement libres. On pose, pour $0 \leq l \leq k$,

$$
\left.\Lambda_{k}^{l}=\sum_{i \geq l} \operatorname{Im}\left(\mathbf{S}_{i}^{(m)}(\mathcal{E}) \otimes_{\mathcal{O}_{X}} \mathbf{S}_{k-i}^{(m)}\right)(\mathcal{F}) \rightarrow \mathbf{S}_{k}^{(m)}(\mathcal{F})\right) .
$$

Les modules $\Lambda_{k}^{l}$ forment une filtration décroissante de $S_{k}^{(m)}(\mathcal{F})$. Les modules $\Lambda_{k}^{0}$ et $\Lambda_{k}^{k}$ sont isomorphes respectivement à $S_{k}^{(m)}(\mathcal{F})$ et $S_{k}^{(m)}(\mathcal{E})$.

Proposition 1.4.1. - Pour tout $l \leq k$, il existe des suites exactes de $\mathcal{O}_{X^{-}}$ modules

$$
0 \rightarrow \Lambda_{k}^{l+1} \rightarrow \Lambda_{k}^{l} \rightarrow S_{l}^{(m)}(\mathcal{E}) \otimes_{\mathcal{O}_{X}} S_{k-l}^{(m)}(\mathcal{G}) \rightarrow 0 .
$$

L'intérêt de cette construction pour nous est le résultat suivant (1.3.7.3 de [14]).

Proposition 1.4.2. - Il existe un isomorphisme canonique de faisceaux de $\mathcal{O}_{X}$-algèbres graduées

$$
\operatorname{gr} . \mathcal{D}_{X}^{(m)} \simeq \mathbf{S}^{(m)}\left(\mathcal{T}_{X}\right)
$$

1.5. Opérateurs différentiels à valeurs dans un faisceau inversible. - Soit $\mathcal{L}$ un faisceau inversible sur $X$ (on gardera la notation $\mathcal{L}$ pour le $\mathcal{O}_{\mathcal{X}}$-module localement libre de rang 1 obtenu en complétant $\mathcal{L}$ le long de $\pi$ ). On note $\mathcal{D}_{\mathcal{X}}^{\sharp}$ l'un des faisceaux $\widehat{\mathcal{D}}_{\mathcal{X}, \mathbf{Q}}^{(m)}$ ou $\mathcal{D}_{\mathcal{X}, \mathbf{Q}}^{\dagger}$ et

$$
\mathcal{D}_{\mathcal{X}}^{\sharp}(\mathcal{L})=\mathcal{L} \otimes \mathcal{O}_{\mathcal{X}} \mathcal{D}_{\mathcal{X}}^{\sharp} \otimes_{\mathcal{O}_{\mathcal{X}}} \mathcal{L}^{-1} .
$$

Sur $X$ (resp. $\mathcal{X})$ on introduit de même le faisceau $\mathcal{D}_{X}^{(m)}(\mathcal{L})\left(\right.$ resp. $\left.\widehat{\mathcal{D}}_{\mathcal{X}}^{(m)}(\mathcal{L})\right)$. On voit facilement que cette définition généralise la définition 4.4 de [4], du faisceau des opérateurs différentiels à valeurs dans un faisceau inversible $\mathcal{L}$. 
1.6. Faisceau tangent sur un espace homogène. - On termine par le fait classique suivant.

Proposition 1.6.1. - Le faisceau $\mathcal{T}_{X}$ est engendré par ses sections globales.

L'action à gauche de $G \operatorname{sur} X(\operatorname{resp} . G)$ munit $\mathcal{T}_{X}\left(\operatorname{resp} . \mathcal{T}_{G}\right)$ d'une structure de $G$-module équivariant (chapitre 1 de [19]). En particulier, le groupe abélien $\Gamma\left(G, \mathcal{T}_{G}\right)$ est un $G$-module. Le module des dérivations invariantes $\Gamma\left(G, \mathcal{T}_{G}\right)^{G}$ s'identifie à $\operatorname{Lie}(G)$ (II 46.5 de [10]). La formation de ce module commute donc aux changements de base. D'après II 46.3 de [10], on dispose d'une application canonique $\operatorname{Lie}(G) \rightarrow \Gamma\left(X, \mathcal{T}_{X}\right)$. On en déduit une application canonique $u:$ $\mathcal{O}_{X} \otimes_{V} \Gamma\left(G, \mathcal{T}_{G}\right)^{G} \rightarrow \mathcal{T}_{X}$

Dans le cas où la base est un corps algébriquement clos, il est bien connu que $u$ est surjectif et que cela résulte du fait que l'action de $G$ sur $X$ est transitive. Rappelons la démonstration dans ce cas. Supposons donc que $S=\operatorname{spec} \bar{l}$ où $\bar{l}$ est un corps algébriquement clos. Notons $e=1_{G} P$, la classe de l'élément neutre de $G$ dans $G / P$. La fibre $i_{e}^{*} \mathcal{T}_{X}$ est un quotient de Lie $(G)$ d'après II 4.2 de [15], ce qui entraîne que $u(e)$ est surjectif. Il suffit de montrer que $u$ est surjectif audessus des points fermés d'après le lemme de Nakayama. Soit $x \in X(\bar{l})$, alors il existe $g \in G(\bar{l})$ tel que $x=g P$. On dispose alors d'opérateurs de translation $\lambda_{g}: k(x)=\bar{l} \simeq k(e)=\bar{l}$ et $\rho_{g}: i_{x}^{*} \mathcal{T}_{X} \simeq i_{e}^{*} \mathcal{T}_{X}$, semi-linéaires par rapport aux $\lambda_{g}$ tels que le diagramme suivant soit commutatif

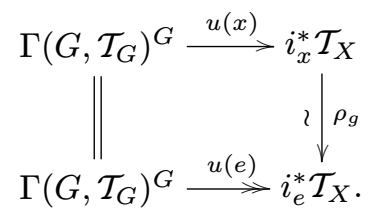

Cela montre que $u(x)$ est surjectif et donc finalement que $u$ est surjectif. Sur une base $S$ générale, il suffit de montrer la surjectivité de $u$ en tout point fermé $s$ de $S$, d'après le lemme de Nakayama. Soient $i_{s}$ l'immersion fermée correspondante à $s, k(s)$ le corps résiduel de $s$ et $\bar{l}$ une clôture algébrique de $k(s)$. Après application de $i_{s}^{*}$, l'application $u$ donne une application $u_{s}$ : $\mathcal{O}_{X_{s}} \otimes_{k(s)} \Gamma\left(G_{s}, \mathcal{T}_{G_{s}}\right)^{G_{s}} \rightarrow \mathcal{T}_{X_{s}}$. Après extension des scalaires à $\bar{l}$, cette flèche est surjective d'après ce qui précède. Par fidèle platitude de $\bar{l}$ sur $k(s)$, la flèche $u_{s}$ est surjective et donc $u$ est surjectif.

Dans la sous-section suivante, on explique pourquoi il suffit de montrer le théorème de Beilinson-Bernstein après extension fidèlement plate de la base. 
1.7. Changements de base fidèlement plats. - Dans cette sous-section, $X$ est un schéma lisse sur $S$, dont le complété formel est $\mathcal{X}$. Soient $m \in \mathbf{N}, \mathcal{D}_{\mathcal{X}}^{\sharp}$ comme en $1.5, V^{\prime}$ un anneau de valuation discrète d'inégales caractéristiques $0, p$, qui est une $V$-algèbre finie fidèlement plate. On introduit aussi $S^{\prime}=\operatorname{spec} V^{\prime}, X^{\prime}=$ $X \times{ }_{S} S^{\prime}, \mathcal{S}^{\prime}=\operatorname{Spf} V^{\prime}, \mathcal{X}^{\prime}=\mathcal{S}^{\prime} \times{ }_{\mathcal{S}} \mathcal{X}$. On dispose alors de la proposition suivante Proposition 1.7.1. - Si $\mathcal{X}^{\prime}$ est $\mathcal{D}_{\mathcal{X}^{\prime}}^{\sharp}$-affine, alors $\mathcal{X}$ est $\mathcal{D}_{\mathcal{X}}^{\sharp}$-affine (resp. si $X^{\prime}$ est $\mathcal{D}_{X^{\prime}}^{(m)}$-affine, alors $X$ est $\mathcal{D}_{X}^{(m)}$-affine).

Démonstration. - Soit $\mathcal{M}$ un $\mathcal{D}_{\mathcal{X}}^{\sharp}$-module cohérent. Il suffit de montrer qu'il est acyclique pour le foncteur $\Gamma$ et engendré par ses sections globales comme $\mathcal{D}_{\mathcal{X}}^{\sharp}$-module. Le $\mathcal{D}_{\mathcal{X}^{\prime}}^{\sharp}$-module $V^{\prime} \otimes_{V} \mathcal{M}$ est cohérent, donc acyclique pour le foncteur $\Gamma$ et engendré par ses sections globales. De plus, par platitude du morphisme $V \rightarrow V^{\prime}$, on a pour tout $n \geq 0$,

$$
H^{n}\left(\mathcal{X}^{\prime}, V^{\prime} \otimes_{V} \mathcal{M}\right)=V^{\prime} \otimes_{V} H^{n}(\mathcal{X}, \mathcal{M})
$$

de sorte que $\forall n \geq 1$, les groupes $H^{n}(\mathcal{X}, \mathcal{M})$ sont nuls. De façon analogue, on a une surjection

$$
\mathcal{D}_{\mathcal{X}^{\prime}}^{\sharp} \otimes_{\Gamma\left(\mathcal{X}^{\prime}, \mathcal{D}_{\mathcal{X}^{\prime}}^{\sharp}\right)} \Gamma\left(\mathcal{X}, V^{\prime} \otimes_{V} \mathcal{M}\right) \rightarrow V^{\prime} \otimes_{V} \mathcal{M},
$$

dont on déduit que $\mathcal{M}$ est engendré par ses sections globales par fidèle platitude de $V^{\prime} \operatorname{sur} V$.

Remarque. On donnera une réciproque à cet énoncé en 2.3.2 en supposant seulement que $V \rightarrow V^{\prime}$ est fini et plat, pour les espaces homogènes, et plus généralement les schémas vérifiant l'hypothèse $(\mathrm{H})$ de 2.

Passons maintenant à la démonstration du théorème principal.

\section{Un critère pour passer du cas algébrique au cas formel}

Le théorème d'annulation va provenir d'un énoncé plus général sur des schémas projectifs sur $S$, sur lesquels le faisceau structural est acyclique et dont le faisceau tangent est engendré par ses sections globales. Il s'agit de donner un critère pour passer d'un énoncé d'acyclicité pour les $\mathcal{D}$-modules sur un schéma projectif lisse $X_{K}$ sur un corps $p$-adique à un tel énoncé d'acyclicité pour les $\mathcal{D}_{\mathcal{X}, \mathbf{Q}}^{\dagger}$-modules cohérents sur le complété formel d'un modèle entier de $X_{K}$. Dans cette partie, on suppose que $X$ est un schéma projectif lisse vérifiant les hypothèses suivantes $(\mathrm{H})$ :

(i) Le faisceau $\mathcal{O}_{X}$ est acyclique pour le foncteur $\Gamma$.

(ii) Le faisceau tangent $\mathcal{T}_{X}$ est engendré par ses sections globales.

TOME $137-2009-\mathrm{N}^{\mathrm{O}} 2$ 
Soit $\mathcal{L}$ un faisceau inversible sur $X$. On note encore $\mathcal{L}$ le faisceau inversible obtenu à partir de $\mathcal{L}$ sur la complétion formelle $\mathcal{X}$ de $X$ et pour n'importe quel symbole $\sharp$ égal à $(m)$ ou $\dagger$, on introduit comme en 1.5 le faisceau des opérateurs différentiels $\mathcal{D}_{\mathcal{X}}^{\sharp}(\mathcal{L})$ à valeurs dans $\mathcal{L}$ défini par $\mathcal{D}_{\mathcal{X}}^{\sharp}(\mathcal{L})=\mathcal{L} \otimes_{\mathcal{O}_{X}} \mathcal{D}_{\mathcal{X}}^{\sharp} \otimes_{\mathcal{O}_{X}} \mathcal{L}^{-1}$.

L'un des points clefs de la démonstration est d'avoir un résultat de finitude de la torsion des groupes $H^{n}\left(X, \mathcal{D}_{X}^{(m)}(s)\right)$ pour $s \in \mathbf{Z}$ fixé et $n \geq 1$. Pour cela on utilise les techniques de [14] et les techniques de Kashiwara exposées en 1.4 de [16]. L'idée consiste à donner un critère analogue à celui de Kashiwara, à torsion finie près.

Fixons maintenant un faisceau ample inversible $\mathcal{O}_{X}(1)$ sur $X$. Si $\mathcal{E}$ est un $\mathcal{O}_{X}$-module, $\mathcal{E}(s)$ pour $s \in \mathbf{Z}$ désigne

$$
\mathcal{E}(s)=\mathcal{E} \otimes_{\mathcal{O}_{X}}\left(\mathcal{O}_{X}(1)\right)^{\otimes s} .
$$

Tout $\mathcal{O}_{X}$-module cohérent est quotient d'un faisceau du type $\mathcal{O}_{X}(-r)^{a}$, avec $a, r \in \mathbf{N}$. De plus, il existe $U \in \mathbf{N}$, tel que pour tout $u \geq U$, le faisceau $\mathcal{O}_{X}(u)$ est engendré par ses sections globales au sens où la flèche suivante est surjective

$$
\mathcal{O}_{X} \otimes_{V} \Gamma\left(X, \mathcal{O}_{X}(u)\right) \rightarrow \mathcal{O}_{X}(u),
$$

et est acyclique pour le foncteur $\Gamma$.

Ces propriétés ainsi que les propriétés cohomologiques du faisceau structural $\mathcal{O}_{X}$ seront essentielles dans ce qui suit.

Soit $\mathcal{L}$ un $\mathcal{O}_{X}$-module localement libre de rang 1 (induisant $\mathcal{L}$ sur $X_{K}$ et $\mathcal{L}$ sur $\mathcal{X}$ ). L'objet de cette section est de montrer le théorème suivant.

ThÉORÈme 2.1. - Soit $X$ un schéma lisse vérifiant les hypothèses $(H), \mathcal{X}$ le schéma formel associé, on a l'énoncé suivant : si $X_{K}$ est $\mathcal{D}_{X_{K}}$-affine (resp. $\mathcal{D}_{X_{K}}(\mathcal{L})$-affine), alors $\mathcal{X}$ est $\mathcal{D}_{\mathcal{X}, \mathbf{Q}}^{\sharp}$-affine (resp. $\mathcal{D}_{\mathcal{X}, \mathbf{Q}}^{\sharp}(\mathcal{L})$-affine).

Ce théorème sera démontré en 2.3.5 et en 2.3.7. En particulier, le complété formel $\mathcal{X}$ d'une variété de drapeaux est $\mathcal{D}_{\mathcal{X}, \mathbf{Q}}^{\sharp}$-affine (resp. $\mathcal{D}_{\mathcal{X}, \mathbf{Q}}^{\sharp}(\lambda)$-affine pour $\lambda$ un poids dominant et régulier).

L'énoncé pour les $\widehat{\mathcal{D}}_{\mathcal{X}, \mathbf{Q}}^{(m)}$-modules repose sur la structure de l'algèbre graduée gr. $\mathcal{D}_{X}^{(m)}$ et sur les résultats classiques de la cohomologie des faisceaux cohérents sur un schéma projectif. On remarquera que si $\mathcal{L}$ est un $\mathcal{O}_{X}$-module inversible,

$$
\operatorname{gr}_{\bullet} \mathcal{D}_{X}^{(m)}(\mathcal{L}) \simeq \mathcal{L} \otimes_{\mathcal{O}_{X}} \operatorname{gr} \mathcal{D}_{X}^{(m)} \otimes \mathcal{O}_{X} \mathcal{L}^{-1}
$$

et donc $\operatorname{gr} \mathcal{D}_{X}^{(m)}(\mathcal{L}) \simeq \operatorname{gr} \mathcal{D}_{X}^{(m)}$ puisque l'algèbre graduée gr. $\mathcal{D}_{X}^{(m)}$ est commutative. Grâce à cette remarque, le lecteur se rendra compte que la démonstration du théorème est la même dans le cas de $\widehat{\mathcal{D}}_{\mathcal{X}, \mathbf{Q}}^{(m)}\left(\right.$ resp. $\left.\mathcal{D}_{\mathcal{X}, \mathbf{Q}}^{\dagger}\right)$ et de $\widehat{\mathcal{D}}_{\mathcal{X}, \mathbf{Q}}^{(m)}(\mathcal{L})$ (resp. $\mathcal{D}_{\mathcal{X}, \mathbf{Q}}^{\dagger}(\mathcal{L})$ ). Pour éviter d'alourdir les notations, nous ferons la démonstration pour le cas de $\widehat{\mathcal{D}}_{\mathcal{X}, \mathbf{Q}}^{(m)}\left(\operatorname{resp} . \mathcal{D}_{\mathcal{X}, \mathbf{Q}}^{\dagger}\right)$. 
2.2. Résultats à un niveau fini. - Dans cette partie, $m$ est fixé. Le premier résultat consiste à établir que si $\mathcal{M}$ est un $\mathcal{D}_{X}^{(m)}$-module cohérent, alors $\mathcal{M}(r)$ est acyclique pour le foncteur $\Gamma(X,$.$) pourvu que r$ soit assez grand. Ce résultat repose sur un résultat analogue pour les modules sur l'algèbre graduée gr $\mathcal{D}_{X}^{(m)}$ et pour les $\mathcal{O}_{X}$-modules. Pour l'algèbre graduée $\operatorname{gr} \bullet \mathcal{D}_{X}^{(m)}$, nous avons en effet la proposition suivante.

Proposition 2.2.1. - Il existe $r_{0} \in \mathbf{N}$ tel que $\forall r \geq r_{0}, \forall n \geq 1$, $H^{n}\left(X, \operatorname{gr}_{\bullet} \mathcal{D}_{X}^{(m)}(r)\right)=0$.

En particulier, pour tous $r \geq r_{0}, n \geq 1, t \geq 0$, les groupes $H^{n}\left(X, \operatorname{gr}_{t} \mathcal{D}_{X}^{(m)}(r)\right)$ sont nuls.

Démonstration. - D'après $(\mathrm{H})$, il existe une surjection $\mathcal{O}_{X}^{a} \rightarrow \mathcal{T}_{X}$, d'où on déduit (1.4.1) un morphisme surjectif de faisceaux cohérents d'algèbres graduées

$$
\mathcal{C}=\mathbf{S}^{(m)}\left(\mathcal{O}_{X}^{a}\right) \rightarrow \mathbf{S}^{(m)}\left(\mathcal{T}_{X}\right),
$$

et cette dernière algèbre graduée s'identifie à l'algèbre graduée $g r \mathcal{D}_{X}^{(m)}(1.4 .2)$. Il suffit donc de montrer l'assertion pour tout $\mathcal{C}$-module cohérent $\mathcal{E}$. Comme $X$ est noethérien, et que $\mathcal{E}$ est un $\mathcal{O}_{X}$-module quasi-cohérent, $\mathcal{E}$ est limite inductive de ses sous $\mathcal{O}_{X}$-modules cohérents $\mathcal{E}_{i}$ pour $i \in I$. Comme $\mathcal{E}$ est un $\mathcal{C}$-module cohérent et que le faisceau d'algèbres $\mathcal{C}$ est à sections noethériennes sur les ouverts affines, il existe une surjection $\mathcal{C}$-linéaire

$$
\mathcal{C} \otimes \mathcal{O}_{X} \mathcal{E}_{i} \rightarrow \mathcal{E}
$$

et donc une surjection $\mathcal{C}$-linéaire

$$
\mathcal{C}\left(s_{0}\right)^{a_{0}} \rightarrow \mathcal{E}
$$

avec $a_{0} \in \mathbf{N}$ et $s_{0} \in \mathbf{Z}$. Si $\mathcal{E}$ est gradué, on peut faire en sorte que la surjection soit graduée mais cela ne sera pas important ici. Soient $r \geq \max \left\{U, U-s_{0}\right\}$ et $\mathcal{E}_{0}=\mathcal{C}\left(s_{0}\right)^{a_{0}}$, les groupes $H^{n}\left(X, \mathcal{E}_{0}(r)\right)$ sont nuls pour tout $n \geq 1$. Soit $\mathcal{E}^{\prime}$ le $\mathcal{C}$-module cohérent qui est le noyau de la surjection $\mathcal{E}_{0} \rightarrow \mathcal{E}$. On considère $\left(a_{n}\right)$ l'assertion suivante : pour tout $\mathcal{C}$-module cohérent $\mathcal{E}$, il existe $r_{0} \geq \max \{U, U-$ $\left.s_{0}\right\}$, tel que $\forall r \geq r_{0}, \forall n \leq k$, les groupes $H^{k}(X, \mathcal{E}(r))$ sont nuls. L'assertion $\left(a_{N+1}\right)$ est vraie par les théorèmes généraux. Pour tout $r \geq \max \left\{U, U-s_{0}\right\}$, et tout $n \in \mathbf{N}^{*}$, on a des suites exactes $0=H^{n}\left(X, \mathcal{E}_{0}(r)\right) \rightarrow H^{n}(X, \mathcal{E}(r)) \rightarrow$ $H^{n+1}\left(X, \mathcal{E}^{\prime}(r)\right)$, et l'assertion $\left(a_{n+1}\right)$ appliquée à $\mathcal{E}^{\prime}$ entraîne l'assertion $\left(a_{n}\right)$ pour $\mathcal{E}$ et la proposition.

On en déduit le corollaire

Corollaire 2.2.2. - (i) $\forall r \geq r_{0}, \forall n \geq 1, \forall t \geq 0, H^{n}\left(X, \mathcal{D}_{X, t}^{(m)}(r)\right)=0$,

(ii) $\forall r \geq r_{0}, \forall n \geq 1, H^{n}\left(X, \mathcal{D}_{X}^{(m)}(r)\right)=0$, 
(iii) Pour tout $\mathcal{D}_{X}^{(m)}$-module cohérent $\mathcal{M}$, il existe $r_{1} \in \mathbf{N}$, tel que $\forall r \geq r_{1}$, $\forall n \geq 1, H^{n}(X, \mathcal{M}(r))=0$.

Démonstration. - Le (ii) résulte du (i) par passage à la limite inductive. On montre le (i) par récurrence sur $t$. Pour $t=0, \mathcal{D}_{X, 0}^{(m)}(r)=\mathcal{O}_{X}(r)$, qui est acyclique pour le foncteur $\Gamma$ car $r \geq r_{0} \geq U$. Pour tout $t \geq 1$, et tout $r \geq r_{0}$, on dispose de suites exactes courtes

$$
0 \rightarrow \mathcal{D}_{X, t-1}^{(m)}(r) \rightarrow \mathcal{D}_{X, t}^{(m)}(r) \rightarrow \operatorname{gr}_{t} \mathcal{D}_{X}^{(m)}(r) \rightarrow 0 .
$$

Comme le faisceau $\operatorname{gr}_{t} \mathcal{D}_{X}^{(m)}(r)$ est acyclique pour $\Gamma$ d'après la proposition précédente, on voit par récurrence sur $t$ qu'il en est de même pour les faisceaux $\mathcal{D}_{X, t}^{(m)}(r)$ après application de la suite exacte longue de cohomologie pour $\Gamma$.

Pour le (iii), on remarque, en procédant comme en 2.2.1, que $\mathcal{M}$ est quotient d'un module $\mathcal{E}_{0}$ du type suivant $\mathcal{E}_{0}=\mathcal{D}_{X}^{(m)}\left(s_{0}\right)^{a_{0}}$, avec $s_{0} \in \mathbf{Z}$ et $a_{0} \in \mathbf{N}$. En utilisant le même argument qu'en 2.2.1, on voit que le (iii) résulte du (ii).

Dans la suite de cette sous-section, on se place sous les hypothèses du théorème et on suppose que $X_{K}$ est $\mathcal{D}_{X_{K}}$-affine.

Le point clé de la démonstration est le résultat suivant, qui concerne la cohomologie des faisceaux $\mathcal{D}_{X}^{(m)}(s)$ pour $s \in \mathbf{Z}$.

Proposition 2.2.3. - Soit $s \in \mathbf{Z}$. Alors $\forall n \geq 1, H^{n}\left(X, \mathcal{D}_{X}^{(m)}(s)\right)$ est un groupe de torsion et cette torsion est bornée.

Démonstration. - Le faisceau $\mathcal{D}_{X, \mathbf{Q}}^{(m)}(s)$ est un $\mathcal{D}_{X_{K}}$-module cohérent, donc pour $n \geq 1$ les groupes $H^{n}\left(X_{K}, \mathcal{D}_{X, \mathbf{Q}}^{(m)}(s)\right)$ sont nuls par hypothèse, et sont égaux à $H^{n}\left(X, \mathcal{D}_{X}^{(m)}(s)\right) \otimes_{V} K$, par commutation de la cohomologie à la limite inductive, de sorte que les groupes $H^{n}\left(X, \mathcal{D}_{X}^{(m)}(s)\right)$ sont de torsion pour $n \geq 1$. Pour voir que la torsion est bornée, on s'inspire des arguments de 1.4 [16].

Fixons $u \geq \max \left\{r_{0}-s, U\right\}$. Commençons par remarquer qu'on a une section $\tilde{\tau}, \mathcal{D}_{X, \mathbf{Q}}$-linéaire à gauche, à la surjection canonique $\tilde{\sigma}$

$$
\mathcal{D}_{X, \mathbf{Q}} \otimes_{\mathcal{O}_{X}} \mathcal{O}_{X_{K}}(-u) \otimes_{K} \Gamma\left(X, \mathcal{O}_{X}(u)\right) \underset{\tilde{\tau}}{\underset{\tau}{\gtrless}} \underset{\tilde{\sigma}_{X, \mathbf{Q}}}{\longrightarrow}
$$

On part en effet de la surjection canonique

$$
\mathcal{D}_{X, \mathbf{Q}} \otimes_{K} \Gamma\left(X_{K}, \mathcal{O}_{X_{K}}(u)\right) \rightarrow \mathcal{D}_{X, \mathbf{Q}} \otimes_{\mathcal{O}_{X_{K}}} \mathcal{O}_{X_{K}}(u) .
$$

Comme le foncteur $\Gamma$ est exact sur la catégorie des $\mathcal{D}_{X, \mathbf{Q}}$-modules cohérents, on trouve une surjection

$$
\Gamma\left(X, \mathcal{D}_{X, \mathbf{Q}}(-u)\right) \otimes_{K} \Gamma\left(X, \mathcal{O}_{X_{K}}(u)\right) \rightarrow \Gamma\left(X, \mathcal{D}_{X, \mathbf{Q}}\right),
$$


dont on trouve une section $\tilde{\tau}, \mathcal{D}_{X, \mathbf{Q}^{-}}$linéaire à gauche, en relevant $1 \in$ $\Gamma\left(X, \mathcal{D}_{X, \mathbf{Q}}\right)$. Observons maintenant qu'il existe $i \in \mathbf{N}$ tel que $\pi^{i} \tilde{\tau}(1) \in$ $\Gamma\left(X, \mathcal{D}_{X}^{(m)}(-u)\right) \otimes_{V} \Gamma\left(X, \mathcal{O}_{X}(u)\right)$. Définissons $\bar{\tau}$ comme l'unique application $\mathcal{D}_{X}^{(m)}$-linéaire $\mathcal{D}_{X}^{(m)} \rightarrow \mathcal{D}_{X}^{(m)} \otimes_{\mathcal{O}_{X}} \mathcal{O}_{X}(-u) \otimes_{V} \Gamma\left(X, \mathcal{O}_{X}(u)\right)$ définie par $\bar{\tau}(1)=\pi^{i} \tilde{\tau}(1)$. En particulier, le conoyau de l'application suivante $\tilde{\sigma}_{m}$ est annulé par $\pi^{i}$

$$
\mathcal{D}_{X}^{(m)} \otimes_{V} \Gamma\left(X, \mathcal{O}_{X}(u)\right) \rightarrow \mathcal{D}_{X}^{(m)}(u) .
$$

Par construction, on a alors

$$
\tilde{\sigma}_{m} \circ \bar{\tau}_{m}=\pi^{i} i d_{\mathcal{D}_{X}^{(m)}} .
$$

Si on dualise ce diagramme, i.e. en appliquant $\mathcal{H o m}_{\mathcal{D}_{X}^{(m)}}\left(., \mathcal{D}_{X}^{(m)}\right)$, on a l'identification suivante pour le terme de gauche

$$
\mathcal{H o m}_{\mathcal{D}_{X}^{(m)}}\left(\mathcal{D}_{X}^{(m)} \otimes_{V} \Gamma\left(X, \mathcal{O}_{X}(u)\right), \mathcal{D}_{X}^{(m)}\right) \simeq \mathcal{D}_{X}^{(m)} \otimes_{V} \Gamma\left(X, \mathcal{O}_{X}(u)\right)^{*},
$$

où $\Gamma\left(X, \mathcal{O}_{X}(u)\right)^{*}=\operatorname{Hom}_{V}\left(\Gamma\left(X, \mathcal{O}_{X}(u)\right), V\right)$. Si $\left(e_{i}\right)_{1 \leq i \leq r}$ est une base de $\Gamma\left(X, \mathcal{O}_{X}(u)\right)$ et $\left(e_{i}^{*}\right)_{1 \leq i \leq r}$ est la base duale de $\Gamma\left(X, \mathcal{O}_{X}(u)\right)^{*}$, l'isomorphisme précédent envoie un homomorphisme $\mathcal{D}_{X}^{(m)}$-linéaire $u$ du 1 er terme sur $\sum_{i=1}^{r} u\left(e_{i}\right) \otimes e_{i}^{*}$ et est $\mathcal{D}_{X}^{(m)}$-linéaire à droite de façon évidente. En dualisant, le terme de droite du diagramme est isomorphe à $\mathcal{D}_{X}^{(m)}(-u)$. En tensorisant ensuite par $\mathcal{O}_{X}(u+s)$, on trouve donc le diagramme suivant de $\mathcal{D}_{X}^{(m)}$-modules à droite

$$
\mathcal{D}_{X}^{(m)}(s) \underset{<\bar{\tau}_{m}}{\stackrel{\sigma_{m}}{\rightleftarrows}} \mathcal{D}_{X}^{(m)}(u+s) \otimes_{V} \Gamma\left(X, \mathcal{O}_{X}(u)\right)^{*},
$$

et on a la relation

$$
\tau_{m} \circ \sigma_{m}=\pi^{i} i d_{\mathcal{D}_{X}^{(m)}(s)} .
$$

Considérons maintenant le diagramme commutatif suivant, pour $n \geq 1, t \geq 0$,

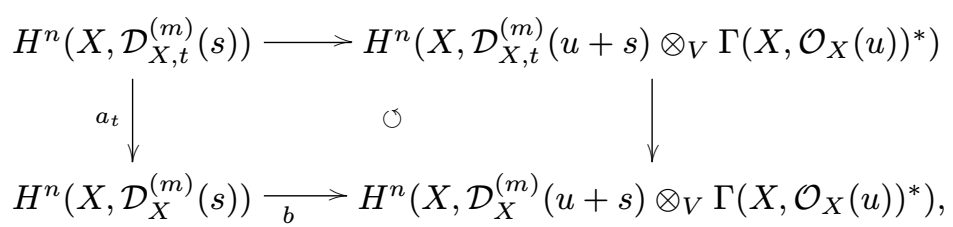

avec $b=H^{n}\left(\sigma_{m}\right)$. Posons aussi $c=H^{n}\left(\tau_{m}\right)$, de sorte que

$$
c \circ b=\pi^{i} i d_{H^{n}\left(X, \mathcal{D}_{X}^{(m)}(s)\right)} .
$$

On a choisi $u$ pour que les deux termes de la colonne de droite du diagramme soient nuls, ce qui implique que $b \circ a_{t}=0$ et donc, en composant avec $c$, que $\pi^{i} a_{t}=0$. En passant à la limite inductive sur $t$, cela nous donne que, pour $n \geq 1$ fixé, $\pi^{i} H^{n}\left(X, \mathcal{D}_{X}^{(m)}(s)\right)=0$ et donc l'énoncé de la proposition. 
On en tire le corollaire suivant.

Corollaire 2.2.4. - Soit $\mathcal{M}$ un $\mathcal{D}_{X}^{(m)}$-module cohérent, alors $\forall n \geq 1$, $H^{n}(X, \mathcal{M})$ est de torsion bornée.

Démonstration. - Puisque $\mathcal{D}_{X}^{(m)}$ est à sections noethériennes sur les affines, on peut procéder comme en 2.2.1 et $\mathcal{M}$ admet une résolution $\mathcal{D}_{X}^{(m)}$-linéaire de longueur $\geq N$ par des modules sommes directe de modules du type $\mathcal{D}_{X}^{(m)}(s)$. En procédant comme en 2.2.1, on voit que les groupes $H^{n}(X, \mathcal{M})$ sont de torsion bornée pour $n \geq 1$.

Contrairement aux résultats de la partie 4 de [14], nous ne pouvons pas donner d'énoncé de finitude des sections globales de $\mathcal{D}_{X}^{(m)}$. Cela vient du fait qu'on ne sait pas si $\Gamma\left(X, \operatorname{gr}_{\bullet} \mathcal{D}_{X}^{(m)}\right)$ est finie sur gr $\Gamma^{\prime}\left(X, \mathcal{D}_{X}^{(m)}\right)$ en général. Sous les hypothèses $(\mathrm{H})$, on a le résultat de finitude suivant sur la cohomologie des $\mathbf{S}^{(m)}\left(\mathcal{T}_{X}\right)$-modules. Reprenons les notations de 2.2.1.

Proposition 2.2.5. - L'algèbre $\Gamma\left(X, \mathbf{S}^{(m)}\left(\mathcal{T}_{X}\right)\right)$ est noethérienne. De plus, si $\mathcal{E}$ est un $\mathbf{S}^{(m)}\left(\mathcal{T}_{X}\right)$-module cohérent, pour tout $n \in \mathbf{N}$, le module $H^{n}(X, \mathcal{E})$ est de type fini sur $\Gamma\left(X, \mathbf{S}^{(m)}\left(\mathcal{T}_{X}\right)\right)$.

Démonstration. - Fixons un plongement projectif $i^{\prime}: X \hookrightarrow Y=\mathbf{P}_{V}^{N}$ tel que $\mathcal{O}_{X}(1)=i^{\prime *} \mathcal{O}_{Y}(1)$. Par hypothèse $(\mathrm{H})$, le faisceau $\mathbf{S}^{(m)}\left(\mathcal{T}_{X}\right)$ est un $\mathcal{C}$-module cohérent. Posons $\mathcal{C}^{\prime}=\mathbf{S}^{(m)}\left(\mathcal{O}_{Y}^{a}\right)$. Alors le faisceau $i_{*}^{\prime} \mathbf{S}^{(m)}\left(\mathcal{T}_{X}\right)$ est un $\mathcal{C}^{\prime}$-module cohérent. Le morphisme $i^{\prime}$ est fini, de sorte qu'il suffit de montrer que $C^{\prime}=$ $\Gamma\left(Y, \mathcal{C}^{\prime}\right)$ est une algèbre noethérienne et que si $\mathcal{F}$ est un $\mathcal{C}^{\prime}$-module cohérent, les modules $H^{n}(Y, \mathcal{F})$ sont de type fini sur $C^{\prime}$. Remarquons que $C^{\prime}=\mathbf{S}^{(m)}\left(V^{a}\right)$ est une $V$-algèbre libre de type fini (1.4) et est donc noethérienne. Par le même argument que celui qui est utilisé en 2.2.1, il suffit de montrer que les modules $H^{n}\left(Y, \mathcal{C}^{\prime}(s)\right)$ sont de type fini sur $C^{\prime}$ pour tout $s \in \mathbf{Z}$ et tout $n \in \mathbf{N}$. Comme $C^{\prime}$ est un $V$-module libre, on a

$$
C^{\prime} \otimes_{V} H^{n}\left(Y, \mathcal{O}_{Y}(s)\right) \simeq H^{n}\left(Y, C^{\prime} \otimes_{V} \mathcal{O}_{Y}(s)\right) \simeq H^{n}\left(Y, \mathcal{C}^{\prime}(s)\right)
$$

Comme les groupes $H^{n}\left(Y, \mathcal{O}_{Y}(s)\right)$ sont des $V$-modules de type fini, cela donne le fait que les $C^{\prime}$-modules $H^{n}(Y, \mathcal{F})$ sont de type fini pour tout $\mathcal{C}^{\prime}$-module cohérent $\mathcal{F}$. C'est en particulier le cas pour $\Gamma\left(X, \mathbf{S}^{(m)}\left(\mathcal{T}_{X}\right)\right.$ ), qui est donc une algèbre noethérienne.

Il s'agit désormais de passer au cas du schéma formel $\mathcal{X}$. 
2.3. Passage au schéma formel. - On suppose dans toute cette sous-section que $X$ est un $S$-schéma vérifiant l'hypothèse $(\mathrm{H})$. On commence par montrer que la catégorie des $\widehat{\mathcal{D}}_{\mathcal{X}}^{(m)}$-modules cohérents est engendrée par les modules du type $\widehat{\mathcal{D}}_{\mathcal{X}}^{(m)}(-r)$ pour $r \in \mathbf{Z}$. Cela correspond à la proposition 3.5 de [14]. La démonstration est identique et suit de 2.2.2.

Proposition 2.3.1. - Soit $\mathcal{M}$ un $\widehat{\mathcal{D}}_{\mathcal{X}}^{(m)}$-module cohérent (resp. un $\mathcal{D}_{\mathcal{X}, \mathbf{Q}^{-}}^{\dagger}$ module cohérent).

(i) Il existe $r_{2} \in \mathbf{N}$, tel que $\forall r \geq r_{2}, \forall n \geq 1, H^{n}(\mathcal{X}, \mathcal{M}(r))=0$.

(ii) Il existe $(a, r) \in \mathbf{N}^{2}$ et une surjection $\widehat{\mathcal{D}}_{\mathcal{X}}^{(m)}$-linéaire $\left(\widehat{\mathcal{D}}_{\mathcal{X}}^{(m)}(-r)\right)^{a} \rightarrow$ $\mathcal{M}$ (resp. $(a, b, r, s) \in \mathbf{N}^{4}$ et une résolution à 2 termes $\mathcal{D}_{\mathcal{X}, \mathbf{Q}}^{\dagger}$-linéaire $\left.\left(\mathcal{D}_{\mathcal{X}, \mathbf{Q}}^{\dagger}(-s)\right)^{b} \rightarrow\left(\mathcal{D}_{\mathcal{X}, \mathbf{Q}}^{\dagger}(-r)\right)^{a} \rightarrow \mathcal{M} \rightarrow 0\right)$.

Comme corollaire, on en déduit une réciproque à 1.7.1. Reprenons les notations de cet énoncé en supposant seulement que le morphisme $V \rightarrow V^{\prime}$ est fini et plat. Alors, on a

Corollaire 2.3.2. - Si $\mathcal{X}$ est $\mathcal{D}_{\mathcal{X}}$-affine, $\mathcal{X}^{\prime}$ est $\mathcal{D}_{\mathcal{X}^{\prime}}$-affine.

Démonstration. - Il est clair que $X^{\prime}$ vérifie $(\mathrm{H})$. Soit $\mathcal{M}$ un $\mathcal{D}_{\mathcal{X}^{\prime}}$-module cohérent. Ce module admet une résolution de longueur arbitrairement grande par des modules du type $\mathcal{D}_{\mathcal{X}^{\prime}}(-r)^{a}$. Par le même argument qu'en 2.2.1, il suffit de montrer que les modules $\mathcal{D}_{\mathcal{X}^{\prime}}(-r)$ sont acycliques pour $\Gamma$ pour vérifier qu'il en est de même pour $\mathcal{M}$. Or, $\mathcal{D}_{\mathcal{X}^{\prime}}(-r)=V^{\prime} \otimes_{V} \mathcal{D}_{\mathcal{X}}(-r)$, et comme $V \rightarrow V^{\prime}$ est plat, on a, pour tout $n \geq 0$,

$$
H^{n}\left(\mathcal{X}^{\prime}, \mathcal{D}_{\mathcal{X}^{\prime}}(-r)\right)=V^{\prime} \otimes_{V} H^{n}\left(\mathcal{X}, \mathcal{D}_{\mathcal{X}}(-r)\right),
$$

d'où l'énoncé d'acyclicité. De plus, le faisceau $\mathcal{D}_{\mathcal{X}^{\prime}}(-r)$ qui est obtenu par changement de base à partir de $\mathcal{D}_{\mathcal{X}}(-r)$, est engendré par ses sections globales comme $\mathcal{D}_{\mathcal{X}^{\prime}}$-module. Comme le foncteur $\Gamma$ est exact pour les $\mathcal{D}_{\mathcal{X}^{\prime}}$-modules cohérents, on dispose d'un diagramme commutatif

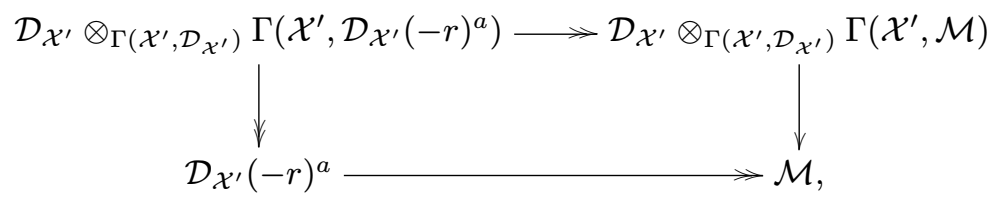

qui montre que $\mathcal{M}$ est engendré par ses sections globales comme $\mathcal{D}_{\mathcal{X}^{\prime}}$-module. 
A partir de maintenant, dans tout le reste de cette sous-section, on suppose que $X_{K}$ est $\mathcal{D}_{X_{K}}$-affine. Grâce à la proposition précédente 2.3.1, on est ramené à contrôler les groupes $H^{n}\left(X, \mathcal{D}_{X}^{(m)}(s)\right)$, en vue de l'énoncé d'acyclicité. Dans la partie 3. de [14], on utilise le fait que les groupes $H^{n}\left(X, \mathcal{D}_{X}^{(m)}(s)\right)$ sont des $V$-modules de type fini de torsion pour $n \geq 1$ et $s \in \mathbf{Z}$. La différence ici est que l'on n'a pas de propriétés de finitude sur $V$, mais on sait que ces groupes sont de torsion bornée d'après 2.2.3. Cependant, le lecteur pourra vérifier que dans la partie 3 de [14], seule la finitude de la torsion des groupes $H^{n}(X, \mathcal{M})$ est utilisée. Cette propriété permet de vérifier des conditions de Mittag-Leffler pour les groupes de cohomologie $H^{n}\left(X_{i}, \mathcal{D}_{X_{i}}^{(m)}(s)\right)$ pour $i$ variable, et permettent des passages à la limite pour la cohomologie. Le résultat suivant se démontre comme la proposition 3.2 de [14] compte tenu de 2.2.3.

Proposition 2.3.3. - Soit $\mathcal{M}$ un $\mathcal{D}_{\mathcal{X}}^{(m)}$-module cohérent et $\widehat{\mathcal{M}}=\lim _{i} \mathcal{M} / \pi^{i+1} \mathcal{M}$. Alors

(i) $\forall n \in \mathbf{N}, H^{n}(\mathcal{X}, \widehat{\mathcal{M}})=\varliminf_{i} H^{n}\left(X_{i}, \mathcal{M} / \pi^{i+1} \mathcal{M}\right)$,

(ii) $\forall n \geq 1, H^{n}(\mathcal{X}, \widehat{\mathcal{M}})=H^{n}(X, \mathcal{M})$.

En particulier, pour $n \geq 1, H^{n}(\mathcal{X}, \widehat{\mathcal{M}})$ est un $V$-module de torsion bornée.

A partir du (ii) de la proposition 2.3.1, on peut procéder comme pour 2.2.4, ce qui donne le résultat de finitude suivant

Proposition 2.3.4. - Soit $\mathcal{M}$ un $\widehat{\mathcal{D}}_{\mathcal{X}}^{(m)}$-module cohérent, alors pour tout $n \geq$ 1 , les groupes $H^{n}(\mathcal{X}, \mathcal{M})$ sont de torsion bornée.

Donnons les conséquences de ces résultats pour les $\widehat{\mathcal{D}}_{\mathcal{X}, \mathbf{Q}}^{(m)}$-modules cohérents. Soit $\mathcal{N}$ un $\widehat{\mathcal{D}}_{\mathcal{X}, \mathbf{Q}}^{(m)}$-module cohérent. Comme l'espace topologique associé à $\mathcal{X}$ est noethérien, il existe d'après 3.4.5 de [3] un $\widehat{\mathcal{D}}_{\mathcal{X}}^{(m)}$-module cohérent $\mathcal{M}$ tel que $\mathcal{N}=\mathcal{M} \otimes_{V} K$. Soit maintenant $\mathcal{N}$ un $\mathcal{D}_{\mathcal{X}, \mathbf{Q}^{-}}^{\dagger}$ module cohérent, d'après 3.6.2 de [3], il existe un $\widehat{\mathcal{D}}_{\mathcal{X}, \mathbf{Q}}^{(m)}$-module cohérent $\mathcal{N}_{0}$ tel que

$$
\mathcal{N} \simeq \mathcal{D}_{\mathcal{X}, \mathbf{Q}}^{\dagger} \otimes_{\widehat{\mathcal{D}}_{\mathcal{X}, \mathbf{Q}}^{(m)}} \mathcal{N}_{0}
$$

Comme la cohomologie commute à la limite inductive sur $\mathcal{X}$, les deux propositions précédentes nous permettent de montrer les énoncés suivants, en procédant comme en 3.5 de [14], et de montrer la partie «acyclicité » de 2.1.

Proposition 2.3.5. - Soit $\mathcal{N}$ un $\widehat{\mathcal{D}}_{\mathcal{X}, \mathbf{Q}}^{(m)}$-module cohérent (resp. un $\mathcal{D}_{\mathcal{X}, \mathbf{Q}^{-}}^{\dagger}$ module cohérent), alors $\forall n \geq 1, H^{n}(X, \mathcal{N})=0$, 
Indiquons comment passer à des énoncés de $\widehat{\mathcal{D}}_{\mathcal{X}, \mathbf{Q}}^{(m)}$-affinité (resp. $\mathcal{D}_{\mathcal{X}, \mathbf{Q}^{-}}^{\dagger}$ affinité). Fixons $u \geq \max \left\{r_{0}, U\right\}$ et reprenons les applications $\tau_{m}$ et $\sigma_{m}$ pour $s=0$ construites lors de la démonstration de 2.2.3. Complétons ces applications, tensorisons par $\mathcal{O}_{\mathcal{X}}(-u)$ et inversons $\pi$, cela nous donne un diagramme

$$
\widehat{\mathcal{D}}_{\mathcal{X}, \mathbf{Q}}^{(m)}(-u)_{\underset{\widehat{\tau}_{m}}{\longrightarrow}}^{\stackrel{\widehat{\sigma}_{m}}{\longrightarrow}} \widehat{\mathcal{D}}_{\mathcal{X}, \mathbf{Q}}^{(m)} \otimes_{V} \Gamma\left(X, \mathcal{O}_{X}(u)\right)^{*}
$$

et l'application $s_{m}=\pi^{-i} \widehat{\tau}_{m}$ est une section $\widehat{\mathcal{D}}_{\mathcal{X}, \mathbf{Q}}^{(m)}$-linéaire de $\widehat{\sigma}_{m}$. On obtient une surjection $\widehat{\mathcal{D}}_{\mathcal{X}, \mathbf{Q}}^{(m)}$-linéaire $\widehat{\mathcal{D}}_{\mathcal{X}, \mathbf{Q}}^{(m)} \otimes_{V} \Gamma\left(\mathcal{X}, \mathcal{O}_{\mathcal{X}}(u)\right)^{*} \rightarrow \widehat{\mathcal{D}}_{\mathcal{X}, \mathbf{Q}}^{(m)}(-u)$. En utilisant la proposition 2.3.1, on trouve ainsi l'énoncé pour les $\widehat{\mathcal{D}}_{\mathcal{X}, \mathbf{Q}}^{(m)}$-modules cohérents. Si $\mathcal{N}$ est un $\mathcal{D}_{\mathcal{X}, \mathbf{Q}}^{\dagger}$-module cohérent, il existe entier $m$, un $\widehat{\mathcal{D}}_{\mathcal{X}, \mathbf{Q}}^{(m)}$-module cohérent $\mathcal{N}_{0}$, tel que

$$
\mathcal{N} \simeq \mathcal{D}_{\mathcal{X}, \mathbf{Q}}^{\dagger} \otimes_{\widehat{\mathcal{D}}_{\mathcal{X}, \mathbf{Q}}^{(m)}} \mathcal{N}_{0}
$$

Pour tout entier $m^{\prime} \geq m$, le module $\widehat{\mathcal{D}}_{\mathcal{X}, \mathbf{Q}}^{\left(m^{\prime}\right)} \otimes_{\widehat{\mathcal{D}}_{\mathcal{X}, \mathbf{Q}}^{(m)}} \mathcal{N}_{0}$ est acyclique pour le foncteur $\Gamma$ par ce qui précède et $\mathcal{N}$ aussi, par commutation de la cohomologie à la limite inductive.

On reprend à partir de maintenant la notation $\mathcal{D}_{\mathcal{X}}^{\sharp}$ introduite en 1.5 . De plus, on note $D^{\sharp}=\Gamma\left(\mathcal{X}, \mathcal{D}_{\mathcal{X}}^{\sharp}\right)$.

Proposition 2.3.6. - Soit $\mathcal{N}$ un $\mathcal{D}_{\mathcal{X}}^{\sharp}$-module cohérent, alors il existe une résolution à deux termes $\mathcal{D}_{\mathcal{X}}^{\sharp}$-linéaire du type suivant

$$
\left(\mathcal{D}_{\mathcal{X}}^{\sharp}\right)^{b} \rightarrow\left(\mathcal{D}_{\mathcal{X}}^{\sharp}\right)^{a} \rightarrow \mathcal{N} \rightarrow 0
$$

Le corollaire qui vient achève la démonstration du théorème 2.1. Rappelons que $X$ est un $S$-schéma vérifiant l'hypothèse cohomologique (H) de 2 .

Corollaire 2.3.7. - Les foncteurs $\Gamma(\mathcal{X},$.$) et \mathcal{D}_{\mathcal{X}}^{\sharp} \otimes_{D^{\sharp}} \cdot$ sont quasi-inverses et induisent une équivalence de catégories entre la catégorie des $D^{\sharp}$-modules à gauche de présentation finie et la catégorie des $\mathcal{D}_{\mathcal{X}}^{\sharp}$-modules à gauche cohérents.

Soit $M$ un $D^{\sharp}$-module de présentation finie et

$$
D^{\sharp^{a}} \rightarrow D^{\sharp^{b}} \rightarrow M \rightarrow 0
$$

une présentation de $M$. En tensorisant cette présentation par $\mathcal{D}_{\mathcal{X}}^{\sharp}$, on trouve une présentation

$$
\mathcal{D}_{\mathcal{X}}^{\sharp b} \rightarrow \mathcal{D}_{\mathcal{X}}^{\sharp a} \rightarrow \mathcal{D}_{\mathcal{X}}^{\sharp} \otimes_{D^{\sharp}} M \rightarrow 0 .
$$

TOME $137-2009-\mathrm{N}^{\circ} 2$ 
En particulier, le module $\mathcal{D}_{\mathcal{X}}^{\sharp} \otimes_{D^{\sharp}} M$ est cohérent comme $\mathcal{D}_{\mathcal{X}}^{\sharp}$-module à gauche. Par acyclicité du foncteur $\Gamma(\mathcal{X},$.$) pour les \mathcal{D}_{\mathcal{X}}^{\sharp}$-modules cohérents, on trouve un diagramme dont les deux carrés sont commutatifs

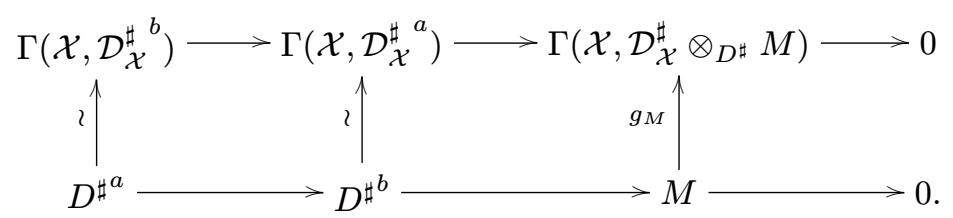

Cela indique que la flèche $g_{M}$ est un isomorphisme.

Partons maintenant d'un $\mathcal{D}_{\mathcal{X}}^{\sharp}$-module cohérent $\mathcal{M}$. D'après la proposition précédente 2.3.6, il existe une résolution

$$
\mathcal{D}_{\mathcal{X}}^{\sharp b} \rightarrow \mathcal{D}_{\mathcal{X}}^{\sharp a} \rightarrow \mathcal{M} \rightarrow 0 .
$$

Comme le foncteur $\Gamma$ est exact, on peut procéder comme précédemment pour voir que la flèche canonique $\mathcal{D}_{\mathcal{X}}^{\sharp} \otimes_{D^{\sharp}} \Gamma(\mathcal{X}, \mathcal{M}) \rightarrow \mathcal{M}$ est un isomorphisme.

Une première application de ce résultat est que l'on peut donner des propriétés de finitude des algèbres de sections globales $D^{\sharp}$.

2.4. Structure des algèbres de sections globales. - On garde les notations de 2.3.7, en supposant toujours que $X$ vérifie $(\mathrm{H})$ et que $X_{K}$ est $\mathcal{D}_{X_{K}}$-affine. Commençons par des énoncés au niveau $m \in \mathbf{N}$, et notons $\widehat{D}_{\mathbf{Q}}^{(m)}=\Gamma\left(\mathcal{X}, \widehat{\mathcal{D}}_{\mathcal{X}, \mathbf{Q}}^{(m)}\right)$. On a la proposition.

Proposition 2.4.1. - (i) Le faisceau $\widehat{\mathcal{D}}_{\mathcal{X}, \mathbf{Q}}^{(m)}$ est un faisceau de $\widehat{D}_{\mathbf{Q}}^{(m)}$-modules à droite plats.

(ii) L'algèbre $\widehat{D}_{\mathbf{Q}}^{(m+1)}$ est une $\widehat{D}_{\mathbf{Q}}^{(m)}$-algèbre plate à droite.

Démonstration. - Commençons par (i). Soit $I$ un idéal à gauche de type fini de $\widehat{D}_{\mathbf{Q}}^{(m)}$. Comme le faisceau $\widehat{\mathcal{D}}_{\mathcal{X}, \mathbf{Q}}^{(m)}$ et à sections noethériennes sur les ouverts affines, le faisceau

$$
\mathcal{I}=\widehat{\mathcal{D}}_{\mathcal{X}, \mathbf{Q}}^{(m)} \otimes_{\widehat{D}_{\mathbf{Q}}^{(m)}} I
$$

est un faisceau de $\widehat{\mathcal{D}}_{\mathcal{X}, \mathbf{Q}^{-m}}^{(m)}$-modules cohérents. Partons de la suite exacte de $\widehat{D}_{\mathbf{Q}}^{(m)}$ modules à gauche de type fini

$$
(E): \quad 0 \rightarrow I \rightarrow \widehat{D}_{\mathbf{Q}}^{(m)} \rightarrow \widehat{D}_{\mathbf{Q}}^{(m)} / I \rightarrow 0 .
$$

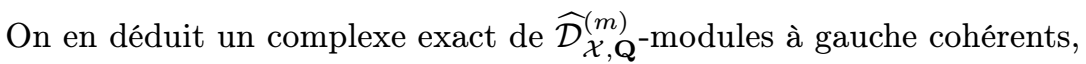

$$
0 \rightarrow \mathcal{T} \rightarrow \mathcal{I} \rightarrow \widehat{\mathcal{D}}_{\mathcal{X}, \mathbf{Q}}^{(m)} \rightarrow \widehat{\mathcal{D}}_{\mathcal{X}, \mathbf{Q}}^{(m)} / \widehat{\mathcal{D}}_{\mathcal{X}, \mathbf{Q}}^{(m)} I \rightarrow 0
$$


avec $\mathcal{T}=\operatorname{Tor}_{\widehat{D}_{\mathbf{Q}}^{(m)}}^{1}\left(\widehat{\mathcal{D}}_{\mathcal{X}, \mathbf{Q}}^{(m)}, \widehat{D}_{\mathbf{Q}}^{(m)} / I\right)$. On peut donc appliquer le foncteur exact $\Gamma$, ce qui donne un diagramme dont tous les carrés sont commutatifs

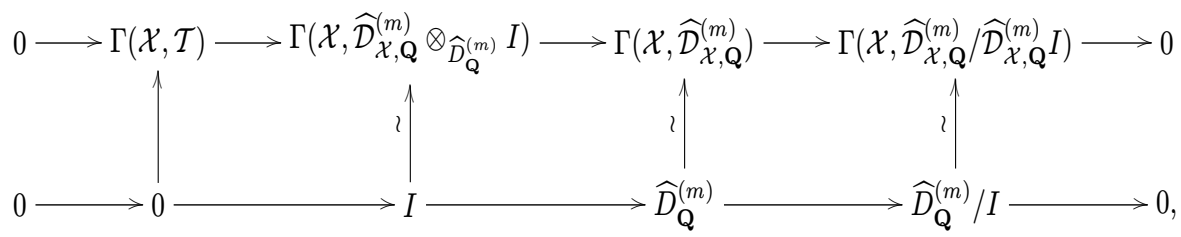

et dont les 3 dernières flèches verticales sont des isomorphismes en vertu de 2.3.7. On en déduit que $\Gamma(\mathcal{X}, \mathcal{T})=0$ et, donc que $\mathcal{T}=0$ puisque $\mathcal{T}$ est un $\widehat{\mathcal{D}}_{\mathcal{X}, \mathbf{Q}}^{(m)}$-module est cohérent.

En conséquence, le faisceau $\widehat{\mathcal{D}}_{\mathcal{X}, \mathbf{Q}}^{(m+1)}$ qui est plat sur $\widehat{\mathcal{D}}_{\mathcal{X}, \mathbf{Q}}^{(m)}$, est aussi plat sur $\widehat{D}_{\mathbf{Q}}^{(m)}$. Pour (ii), tensorisons la suite exacte $(E)$ par $\widehat{\mathcal{D}}_{\mathcal{X}, \mathbf{Q}}^{(m+1)}$ : on trouve une suite exacte de $\widehat{\mathcal{D}}_{\mathcal{X}, \mathbf{Q}}^{(m+1)}$-modules à gauche cohérents

$$
0 \rightarrow \widehat{\mathcal{D}}_{\mathcal{X}, \mathbf{Q}}^{(m+1)} \otimes_{\widehat{D}_{\mathbf{Q}}^{(m)}} \mathcal{I} \rightarrow \widehat{\mathcal{D}}_{\mathcal{X}, \mathbf{Q}}^{(m+1)} \rightarrow \widehat{\mathcal{D}}_{\mathcal{X}, \mathbf{Q}}^{(m+1)} / \widehat{\mathcal{D}}_{\mathcal{X}, \mathbf{Q}}^{(m+1)} I \rightarrow 0
$$

Passons aux sections globales, qui est un foncteur exact et appliquons de nouveau l'équivalence 2.3 .7 : on trouve une suite exacte de $\widehat{D}^{(m+1)}$-modules à gauche

$$
0 \rightarrow \widehat{D}_{\mathbf{Q}}^{(m+1)} \otimes_{\widehat{D}_{\mathbf{Q}}^{(m)}} I \rightarrow \widehat{D}_{\mathbf{Q}}^{(m+1)} \rightarrow \widehat{D}_{\mathbf{Q}}^{(m+1)} / \widehat{D}_{\mathbf{Q}}^{(m+1)} I \rightarrow 0,
$$

qui donne l'énoncé de platitude (ii) cherché.

On en déduit, à partir des propriétés de finitude des faisceaux $\widehat{\mathcal{D}}_{\mathcal{X}, \mathbf{Q}}^{(m)}$ l'énoncé suivant, en notant $D_{\mathbf{Q}}^{\dagger}=\Gamma\left(\mathcal{X}, \mathcal{D}_{\mathcal{X}, \mathbf{Q}}^{\dagger}\right)$.

THÉORÈmE 2.4.2. - (i) L'algèbre $\widehat{D}_{\mathbf{Q}}^{(m)}$ est une K-algèbre noethérienne à gauche.

(ii) L'algèbre $D_{\mathbf{Q}}^{\dagger}$ est une $K$-algèbre cohérente à gauche.

(iii) Le faisceau $\mathcal{D}_{\mathcal{X}, \mathbf{Q}}^{\dagger}$ est un faisceau de $D_{\mathbf{Q}}^{\dagger}$-modules à droite plats.

Démonstration. - Compte tenu de 2.3.3, $\Gamma\left(\mathcal{X}, \widehat{\mathcal{D}}_{\mathcal{X}}^{(m)}\right)$ est obtenu comme complété de $\Gamma\left(X, \mathcal{D}_{X}^{(m)}\right)$, de sorte que $\Gamma\left(X, \widehat{\mathcal{D}}_{\mathcal{X}, \mathbf{Q}}^{(m)}\right)$ est une $K$-algèbre de Banach. L'algèbre $\Gamma\left(\mathcal{X}, \mathcal{D}_{\mathcal{X}}^{\dagger}\right)$ est limite inductive (sur $\left.m\right)$ des algèbres $\Gamma\left(X, \widehat{\mathcal{D}}_{\mathcal{X}}^{(m)}\right)$ et est donc faiblement complète, tout comme $\Gamma\left(\mathcal{X}, \mathcal{D}_{\mathcal{X}, \mathbf{Q}}^{\dagger}\right)$. Montrons maintenant la 
noethérianité de $\widehat{D}_{\mathbf{Q}}^{(m)}$. Soit $I$ un idéal de $\widehat{D}_{\mathbf{Q}}^{(m)}$ et $\left\{I_{i}\right\}_{i \in \Omega}$ un système inductif d'idéaux de type fini de $D$ tels que

$$
I=\underset{i}{\lim _{\longrightarrow}} I_{i} .
$$

Introduisons $\mathcal{I}_{i}=\mathcal{D} \otimes_{D} I_{i} \subset \mathcal{D}$, qui forment une suite croissante d'idéaux d'après l'énoncé de platitude 2.4.1 précédent. Comme le faisceau $\widehat{\mathcal{D}}_{\mathcal{X}, \mathbf{Q}}^{(m)}$ est à sections noethériennes sur les affines et que $\mathcal{X}$ est quasi-compact, il existe $i_{0} \in \Omega$ tel que $\mathcal{I}_{i_{0}}=\mathcal{I}_{j}$ pour tout $j \geq i_{0}$. Ce qui donne, en appliquant de nouveau 2.3.7, $I_{i_{0}}=I_{j}$ pour tout $j \geq i_{0}$, et $I=I_{i_{0}}$ est de type fini.

L'énoncé (ii) vient du fait que $D_{\mathbf{Q}}^{\dagger}$ est limite inductive d'algèbres noethériennes $\widehat{D}_{\mathbf{Q}}^{(m)}$ telle que les extensions $\widehat{D}_{\mathbf{Q}}^{(m)} \rightarrow \widehat{D}_{\mathbf{Q}}^{(m+1)}$ sont plates par 2.4.1.

L'énoncé de platitude (iii) se démontre exacement comme le (i) de 2.4.1, puisque, d'après la cohérence de $D_{\mathbf{Q}}^{\dagger}$, si $I$ est un idéal de type fini de $D_{\mathbf{Q}}^{\dagger}$, il est de présentation finie. En particulier, le faisceau associé $\mathcal{D}_{\mathcal{X}, \mathbf{Q}}^{\dagger} \otimes_{D_{\mathbf{Q}}^{\dagger}} I$ est un $\mathcal{D}_{\mathcal{X}, \mathbf{Q}}^{\dagger}$-module cohérent. Le même argument que celui de (i) de 2.4.1 s'applique alors.

\section{Le théorème de Beilinson-Bernstein arithmétique}

Tout est désormais en place pour le théorème principal de cet article. Reprenons les notations de 1, i.e. $X$ est une variété de drapeaux d'un groupe algébrique semi-simple sur $S$. Le théorème de Kempf de A.4.2, ainsi que 1.6.1 entraînent que $X$ vérifie l'hypothèse cohomologique (H) de 2. Soit $\lambda \in X(T)$ et $\rho$ la demi-somme des racines positives de $G$. Si $\lambda+\rho$ est dominant, le poids correspondant de l'algèbre de Lie $d \lambda+d \rho$ est dominant (A.2). Soit $\mathcal{L}(\lambda)$ le faisceau inversible associé à $\lambda$ comme en A.4. On peut donc appliquer le théorème principal de [2] et $X$ est $\mathcal{D}_{X, \mathbf{Q}}(\mathcal{L}(\lambda))$-affine. Pour simplifier les notations, nous noterons comme en $1.5 \mathcal{D}_{\mathcal{X}}^{\sharp}$ l'un des faisceaux $\widehat{\mathcal{D}}_{\mathcal{X}, \mathbf{Q}}^{(m)}$ ou $\mathcal{D}_{\mathcal{X}, \mathbf{Q}}^{\dagger}$ et $\mathcal{D}_{\mathcal{X}}^{\sharp}(\lambda)=\mathcal{D}_{\mathcal{X}}^{\sharp}(\mathcal{L}(\lambda))$. Appliquons 2.1. On obtient :

ThÉORÈme 3.1. - Soit $\lambda \in X(T)$ tel que $\lambda+\rho$ est dominant et régulier, alors $\mathcal{X}$ est $\mathcal{D}_{\mathcal{X}}^{\sharp}(\lambda)$-affine.

On obtient de plus les résultats suivants sur les algèbres de sections globales sous les hypothèses du théorème précédent.

ThÉORÈme 3.2. - (i) Pour tout entier $m$, la $V$-algèbre $\Gamma\left(\mathcal{X}, \widehat{\mathcal{D}}_{\mathcal{X}, \mathbf{Q}}^{(m)}(\lambda)\right)$ est une $V$-algèbre complète noethérienne à gauche.

(ii) La $V$-algèbre $\Gamma\left(\mathcal{X}, \mathcal{D}_{\mathcal{X}, \mathbf{Q}}^{\dagger}(\lambda)\right)$ est une $V$-algèbre faiblement complète cohérente à gauche. 
Rappelons en appendice quelques considérations sur les liens entre système de racines de $\operatorname{Lie}(G)$ et donnée de racines de $G$ dans notre contexte, utilisées dans l'énoncé du théorème. Nous n'avons pas trouvé de référence pour ces résultats dans notre contexte (pour des groupes algébriques sur une base qui est un anneau de valuation discrète).

\section{Appendice A}

\section{Comparaison de systèmes de racines.}

Dans cette partie, on suppose que $G$ est semi-simple déployé et on note $V[G]=\Gamma\left(G, \mathcal{O}_{G}\right)$. On pourra remplacer $V$ par $\mathbf{Z}$ si $G$ est défini sur $\mathbf{Z}$ et si un tore maximal est déployé sur $\mathbf{Z}$. On peut alors introduire la donnée de racines de ce groupe algébrique. On peut aussi considérer le système de racines de l'algèbre de Lie de $G$, qui sera définie comme dans [15]. On explique ici comment identifier ces données (après avoir tensorisé par $K$ ). Dans le cas complexe, ces résultats sont bien connus. Faute de référence dans notre cas, nous expliquons comment procéder.

A.1. Donnée de racines d'un groupe algébrique et algèbre de distributions. - On note $1_{G}$ l'élément neutre de $G, 1_{G}: \operatorname{spec} V \hookrightarrow G, \varepsilon$ l'application correspondante $: V[G] \rightarrow V[G], i_{G}: G \rightarrow G$ l'application de passage à l'inverse, $\sigma_{G}:$ $V[G] \rightarrow V[G]$ l'application correspondante, $\mu$ l'application produit $G \times G \rightarrow G$ et $\mu^{\sharp}$ l'application correspondante $V[G] \rightarrow V[G] \times V[G]$. Soit $T$ un tore maximal déployé fixé de $G$. La présentation de Jantzen ([15]) est particulièrement bien adaptée à notre cadre. L'algèbre de groupe $V[G]$ se décompose $V[G]=V \bigoplus I_{1}$ où $I_{1}$ est l'idéal d'augmentation de $G$, c'est-à-dire le noyau du morphisme $\varepsilon$. On pose alors

$$
\operatorname{Lie}(G)=\operatorname{Hom}_{V}\left(I_{1} / I_{1}^{2}, V\right) .
$$

Si $G$ est lisse, on introduit $\mathcal{T}_{G}$ le faisceau tangent du groupe $G$. La suite exacte des faisceaux de formes différentielles appliquée à l'immersion fermée $1_{G}$ (voir par exemple chapitre II prop. 7 de [6]) donne que $I_{1} / I_{1}^{2} \simeq 1_{G}^{*} \Omega_{G / V}^{1}$ et en dualisant, cela donne que $\operatorname{Lie}(G) \simeq 1_{G}^{*} \mathcal{T}_{G}$, de sorte que, si $G$ est lisse, $\operatorname{Lie}(G)$ est un $V$-module libre de rang fini, dont la formation commute aux changements de base.

On introduit aussi

$$
\operatorname{Dist}(G)_{n}=\operatorname{Hom}_{V}\left(V[G] / I_{1}^{n+1}, V\right)
$$

et

$$
\operatorname{Dist}(G)=\underset{n}{\lim } \operatorname{Dist}(G)_{n} .
$$


En utilisant l'application $\mu^{\sharp}$, on montre que Dist $(G)$ est une algèbre (cf I 7.7 de [15]) et que cette structure d'algèbre induit une structure de $K$-algèbre de Lie sur Lie $(G) \otimes_{V} K$. On peut montrer, mais cela ne nous sera pas utile ici, que cette algèbre coïncide avec la fibre en $1_{G}$ du faisceau des opérateurs différentiels $\mathcal{D}_{G}^{(0)}$. Après extension de $V$ à $K$, cette algèbre est l'algèbre enveloppante de $\operatorname{Lie}(G)_{K}$.

Soient $X(T)$ le groupe des caractères de $T\left(X(T)=\operatorname{Hom}\left(T, \mathbf{G}_{m}\right)\right), Y(T)$ le groupe des cocaractères de $T\left(Y(T)=\operatorname{Hom}\left(\mathbf{G}_{m}, T\right)\right)$, $R$ l'ensemble des racines de $G$. Les groupes $X(T)$ et $Y(T)$ sont deux $\mathbf{Z}$-modules libres de rang fini et on dispose du crochet de dualité $<,>: X(T) \times Y(T) \rightarrow \mathbf{Z}$. En effet, soient $(\lambda, \mu) \in X(T) \times Y(T)$, alors $\lambda \circ \mu$ définit un élément de $\operatorname{Hom}_{G r}\left(\mathbf{G}_{m}, \mathbf{G}_{m}\right) \simeq \mathbf{Z}$. A un élément $\lambda$ de $X(T)$, on associe un élément inversible de $V[T]$, que l'on notera aussi $\lambda$. Si $M$ est un $T$-module et $\lambda \in X(T)$, et si $\Delta_{m}$ est l'application de comodule $M \rightarrow M \otimes_{V} V[T]$, on note $M_{\lambda}=\left\{m \in M \mid \Delta_{M}(m)=m \otimes \lambda\right\}$. L'action par conjugaison de $T$ sur Lie $(G)$ notée $A d$ se décompose comme d'habitude

$$
\operatorname{Lie}(G)=\operatorname{Lie}(T) \bigoplus_{\alpha \in R} \operatorname{Lie}(G)_{\alpha} .
$$

A chaque $\alpha$, on associe un élément $\alpha^{\vee}$ de $Y(T)$, en procédant comme en II 1.3 de [15]. L'ensemble des éléments $\alpha^{\vee}$ est noté $R^{\vee}$. Le quadruplet $\left(X(T), R, Y(T), R^{\vee}\right)$ associé à la bijection $R \rightarrow R^{\vee}$ et à l'accouplement $<,>$, constitue la donnée de racines de $G$. Comme $G$ est semi-simple, le couple $\left(R, X(T) \otimes_{\mathbf{Z}} K\right)$ est un système de racines sur le corps $K$ (cf chap. 6 de [7]).

A.2. Système de racines de l'algèbre de Lie d'un groupe algébrique. - Soit $\lambda \in$ $X(T)$. L'application tangente $d \lambda$ définit une application $\operatorname{Lie}(T) \rightarrow \operatorname{Lie}\left(\mathbf{G}_{m}\right)$ et donc un élément de $\operatorname{Lie}(T)^{*}$, une fois fixée une coordonnée $t$ de $\mathbf{G}_{m}$. De même l'application tangente d'un élément $\mu$ de $Y(T)$ est une application $d \mu$ : $\operatorname{Lie}\left(\mathbf{G}_{m}\right) \rightarrow \operatorname{Lie}(T)$. Après avoir identifié $\operatorname{Hom}_{\operatorname{Lie}}\left(\operatorname{Lie}\left(\mathbf{G}_{m}\right), \operatorname{Lie}(T)\right)$ à $\operatorname{Lie}(T)$, on peut voir l'élément $d \mu$ comme un élément de $\operatorname{Lie}(T)$, ce que nous ferons dans la suite. On obtient un accouplement canonique $\langle d \lambda, d \mu\rangle$ en composant $d \lambda \circ d \mu \in \operatorname{Hom}_{\text {Lie }}\left(\operatorname{Lie}\left(\mathbf{G}_{m}\right), \operatorname{Lie}\left(\mathbf{G}_{m}\right)\right) \simeq V$. Par construction, on a, après choix d'une coordonnée $t$ sur $\mathbf{G}_{m}:\langle\lambda, \mu\rangle=\langle d \lambda, d \mu\rangle$ (car l'application tangente en $t=1$ de $t \mapsto t^{n}$ est la multiplication par $\left.n\right)$. L'application $X(T) \rightarrow \operatorname{Lie}(T)^{*}$ n'est pas injective en caractéristique $p>0$ (car le caractère $t \mapsto t^{p}$ est envoyé sur 0$)$, mais après tensorisation par $K$ on a un isomorphisme $X(T) \otimes_{\mathbf{Z}} K \simeq \operatorname{Lie}\left(T_{K}\right)^{*}$ $\left(\operatorname{resp} . X(T) \otimes_{\mathbf{z}} k \simeq \operatorname{Lie}\left(T_{k}\right)^{*}\right)$.

Soit $\alpha \in R$, on lui associe $d \alpha \in \operatorname{Lie}(T)^{*}$, qu'on notera $\alpha_{*}$. A la co-racine $\alpha^{\vee}$, on associe de même $d \alpha^{\vee} \in \operatorname{Lie}(T)$, qu'on notera $H_{\alpha} \in \operatorname{Lie}(T)$. On note enfin $R_{*}=\left\{\alpha_{*} \mid \alpha \in R\right\} \subset \operatorname{Lie}(T)^{*}$ et $R_{*}^{\vee}=\left\{H_{\alpha} \mid \alpha \in R\right\} \subset \operatorname{Lie}(T)$.

On définit comme d'habitude $s_{\alpha}: X(T) \rightarrow X(T)$ par $s_{\alpha}(\lambda)=\lambda-\left\langle\lambda, \alpha^{\vee}\right\rangle \alpha$. Les applications $s_{\alpha}$ sont les réflexions associées au système de racines sur $K$ 
$(R, X(T) \otimes \mathbf{z} K)$. On définit de façon analogue pour $h \in \operatorname{Lie}(T)^{*}, \alpha \in R$, $\sigma_{\alpha_{*}}(h)=h-\left\langle h, \alpha_{*}^{\vee}\right\rangle \alpha_{*}$. On vérifie facilement que les applications $\sigma_{\alpha_{*}}$ sont des réflexions. De plus, on a l'égalité, pour $\alpha, \beta \in R, \sigma_{\alpha_{*}}\left(\beta_{*}\right)=\left(s_{\alpha}(\beta)\right)_{*}$ de sorte que les réflexions $\sigma_{\alpha_{*}}$ préservent $R_{*}$. On identifie ainsi les systèmes de racines $(R, X(T) \otimes \mathbf{z} K)$ et $\left(R_{*}, \operatorname{Lie}\left(T_{K}\right)^{*}\right)$.

On remarquera enfin que si $\lambda \in X(T)$ et si $H \in \operatorname{Lie}(T)$, alors $d \lambda(H)=H(\lambda)$. C'est vrai même si le tore $T$ n'est pas déployé. Pour voir cela, on commence par se ramener au cas où le tore est déployé après une extension fidèlement plate de la base. On est alors ramené à montrer cette égalité pour $\lambda$ un caractère de $\mathbf{G}_{m}$, or, dans ce cas, si $\lambda(t)=t^{n}$, et $H=\partial_{1}$ définie par $\partial_{1}(f)=(\partial f / \partial t)(1)$, on a $d \lambda\left(\partial_{1}\right)=\partial_{1}(\lambda)=n$.

Il nous reste maintenant à vérifier que le système de racines $\left(R_{*}, \operatorname{Lie}\left(T_{K}\right)^{*}\right)$ est le système de racines associé à $\operatorname{Lie}(G)$, c'est-à-dire que les racines ainsi obtenues sont celles données par la représentation adjointe de $\operatorname{Lie}(T) \operatorname{sur} \operatorname{Lie}(G)$. Cela fait l'objet de la sous-section suivante.

A.3. Comparaison des actions adjointes. - L'action adjointe $A d$ de $T$ sur $\operatorname{Lie}(G)$ induit une action adjointe ad $\operatorname{de} \operatorname{Lie}(T) \operatorname{sur} \operatorname{Lie}(G)$ (I 7.11 de [15]), et, si $\alpha \in R$, l'action de $H \in \operatorname{Lie}(T)$ sur $v \in \operatorname{Lie}(G)_{\alpha}$ est donnée par $H(v)=H(\alpha) v$. Dans la suite, nous vérifions que cette action de $\operatorname{Lie}(T)$ déduite de l'action de conjugaison par $T$ est bien l'action adjointe induite par le crochet de Lie de $\operatorname{Lie}(G)$. C'est classique sur le corps des nombres complexes mais nous n'avons pas trouvé de réference sur une base plus générale. En particulier, cette assertion est valable sur un corps $k$ de caractéristique $p>0$ (dans ce cas, le lecteur identifiera $V=k=K$ dans les notations). Pour ce faire, nous utilisons des formules sur les algèbres de distibutions citées dans [15]. Nous en déduirons la description de l'action de $\operatorname{Lie}(T)$ sur $\operatorname{Lie}(G)$ en vertu des injections $\operatorname{Lie}(T) \subset \operatorname{Dist}(T)$, $\operatorname{Lie}(G) \subset \operatorname{Dist}(G)$ et $\operatorname{Dist}(T) \subset \operatorname{Dist}(G)$.

Si $H \in \operatorname{Dist}(G)$, on note $\sigma_{G}^{\prime}(H)=H \circ \sigma_{G}$. En I 7.18 (formule (3)) de [15], Jantzen donne le calcul de l'action obtenue de $\operatorname{Dist}(T) \operatorname{sur} \operatorname{Dist}(G)$. L'immersion diagonale $T \hookrightarrow T \times T$ définit sur $\operatorname{Dist}(T)$ une co-multiplication $\Delta_{T}^{\prime}: \operatorname{Dist}(T) \rightarrow$ $\operatorname{Dist}(T) \otimes_{V} \operatorname{Dist}(T)$ définie par $\Delta_{T}^{\prime}(H)=1 \otimes H+H \otimes 1$. Pour des raisons de fonctorialité, la co-multiplication sur $\operatorname{Dist}(T)$ (resp. $\left.\sigma_{T}^{\prime}\right)$ est la restriction à $\operatorname{Dist}(T)$ de la co-multiplication sur $\operatorname{Dist}(G)\left(\operatorname{resp} . \sigma_{G}^{\prime}\right)$. Si $\Delta_{G}^{\prime}(H)=\sum_{i} H_{i} \otimes$ $H_{i}^{\prime}$, Jantzen établit que ad $(H) H^{\prime}=\sum_{i} H_{i} H^{\prime} \sigma_{G}^{\prime}\left(H_{i}^{\prime}\right) \in \operatorname{Dist}(G)$. Comme $H \in$ $\operatorname{Dist}(T)$, on trouve $\operatorname{ad}(H)\left(H^{\prime}\right)=H H^{\prime}+H^{\prime} \sigma_{T}^{\prime}(H)$. Remarquons maintenant que $\sigma_{T}^{\prime}(H)=-H$. En effet, après extension fidèlement plate de la base, on se ramène au cas où le tore $T$ est déployé, et finalement on est ramené au cas où $T=\mathbf{G}_{m}=\operatorname{spec}\left(V\left[t, t^{-1}\right]\right)$. Dans ce cas, Dist $(T)$ est libre de base $\partial_{1}$ défini par 
$\partial_{1}(f)=(\partial f / \partial t)(1)$. On calcule alors

$$
\sigma_{T}^{\prime}\left(\partial_{1}\right)(f)=\frac{\partial\left(f\left(t^{-1}\right)\right)}{\partial t}(1)=\left(-t^{-2}\left(\frac{\partial f}{\partial t}\right)\left(t^{-1}\right)\right)(1)=-\partial_{1}(f) .
$$

Finalement, cela montre que $\operatorname{ad}(H)\left(H^{\prime}\right)=\left[H, H^{\prime}\right]$, de sorte que la décomposition de $\operatorname{Lie}(G)=\operatorname{Lie}(T) \bigoplus_{h_{\alpha} \in R} \operatorname{Lie}(G)_{\alpha}$ est la décomposition définissant le système de racines de $\operatorname{Lie}\left(G_{K}\right)$.

Un corollaire de toutes ces vérifications est qu'on peut identifier le système de racines $\left(R, X\left(T_{K}\right)\right)$ utilisé par Jantzen dans [15] et le système de racines $\left(R_{*}, \operatorname{Lie}\left(T_{K}\right)\right)$. Il reste à choisir un système de racines positifs. On remarquera que Kashiwara dans [16] et Beilinson-Bernstein dans [2] prennent la convention opposée. Nous adopterons ici la convention de Beilinson-Bernstein (et de Jantzen) en imposant que les racines venant du groupe de Borel constituent un système de racines négatives.

A.4. Poids dominants et réguliers. - Le caractère $\lambda$ induit un poids toujours noté $\lambda$ sur $G_{K}$ et sur $G_{k}$. Soient $V_{\lambda}$, resp. $K_{\lambda}$ et resp. $k_{\lambda}$ les représentations de $T$, resp. $T_{K}$ et $T_{k}$ associées à $\lambda$. A ces représentations, on associe les faisceaux $\mathcal{L}(\lambda)$ sur $X$, resp. $\mathcal{L}\left(\lambda_{k}\right)$ sur $X_{k}$ et $\mathcal{L}\left(\lambda_{K}\right)$ sur $X_{K}$, en procédant comme de I 5 . de [15]. Soient $P$ un sous-groupe parabolique du groupe $G$ et $X=G / P$. Sur $X$ on a la description suivante, au-dessus d'un ouvert affine $U$,

$$
\mathcal{L}(\lambda)(U)=\left(V_{\lambda} \otimes_{V} V[U \cdot P]\right)^{T},
$$

où l'action de $T$ sur le produit tensoriel est l'action produit de l'action de $T$ sur $V_{\lambda}$ et de l'action de $T$ par la représentation régulière droite sur les éléments de $V[U \cdot P])$.

Il résulte de I 5.17 de [15] que $i^{*} \mathcal{L}(\lambda) \simeq \mathcal{L}\left(\lambda_{k}\right)$ (resp. $\left.j^{*} \mathcal{L}(\lambda) \simeq \mathcal{L}\left(\lambda_{K}\right)\right)$. D'autre part, le faisceau $\mathcal{L}(\lambda)$ est inversible sur $X$ d'après I 5.16 de [15].

On rappelle les définitions suivantes (II 2.6 de [15]).

DÉFinition A.4.1. - Un poids $\lambda$ de $G_{K}$ est appelé dominant (resp. régulier) si $\forall \alpha^{\vee} \in R^{\vee}$, on $a\left\langle\lambda, \alpha^{\vee}\right\rangle \geq 0$ (resp. $\forall \alpha^{\vee} \in R^{\vee}$, on $a\left\langle\lambda, \alpha^{\vee}\right\rangle>0$ ).

De façon équivalente, $\lambda$ est dominant si et seulement si le module de sections globales $H^{0}\left(X, \mathcal{L}_{K}(\lambda)\right) \neq 0$ et $\lambda$ est régulier si et seulement si le faisceau $\mathcal{L}_{K}(\lambda)$ est ample. Si $\lambda$ est dominant, $H^{0}\left(X, \mathcal{L}_{K}(\lambda)\right)$ est la représentation irréductible de $G_{K}$ de plus haut poids $\lambda$ (à isomorphisme près). Nous aurons besoin d'une variante sur $X$ du théorème de Kempf (II 4.5 de [15]).

Proposition A.4.2. - Si $\lambda$ est dominant, alors $\forall n \geq 1, H^{n}(X, \mathcal{L}(\lambda))=0$. 
Le résultat est classique pour $\mathcal{L}\left(\lambda_{K}\right)$ et $\mathcal{L}\left(\lambda_{k}\right)$ sur $X_{K}$ et $X_{k}$ respectivement, de sorte que $i^{*} \mathcal{L}(\lambda)$ et $j^{*} \mathcal{L}(\lambda)$ sont acycliques pour le foncteur sections globales. Comme la cohomologie commute à la limite inductive sur un schéma noethérien, pour tout $n \in \mathbf{N}, H^{n}\left(X_{K}, \mathcal{L}\left(\lambda_{K}\right)\right)=K \otimes_{V} H^{n}(X, \mathcal{L}(\lambda))$. Comme ces groupes sont nuls pour $i \geq 1$, on voit que les groupes $H^{n}(X, \mathcal{L}(\lambda))$ sont de torsion et donc de torsion bornée car ce sont des $V$-modules de type fini par le théorème de finitude pour les morphismes propres ([11]). La suite exacte longue de cohomologie associée à la suite exacte courte

$$
0 \rightarrow \mathcal{L}(\lambda) \stackrel{\cdot \pi}{\rightarrow} \mathcal{L}(\lambda) \rightarrow i_{*} \mathcal{L}\left(\lambda_{k}\right) \rightarrow 0,
$$

donne des surjections, pour tout $n \geq 1$

$$
\cdot \pi: H^{n}(X, \mathcal{L}(\lambda)) \rightarrow H^{n}(X, \mathcal{L}(\lambda)),
$$

ce qui montre finalement que ces groupes sont nuls puisqu'ils sont de torsion bornée.

\section{BIBLIOGRAPHIE}

[1] S. AnANTHARAmAN - «Schémas en groupes, espaces homogènes et espaces algébriques sur une base de dimension 1 », in Sur les groupes algébriques, Soc. Math. France, 1973, p. 5-79. Bull. Soc. Math. France, Mém. 33.

[2] A. BeĬlinson \& J. Bernstein - «Localisation de $g$-modules », $C . R$. Acad. Sci. Paris Sér. I Math. 292 (1981), p. 15-18.

[3] P. Berthelot - « $\mathscr{D}$-modules arithmétiques. I. Opérateurs différentiels de niveau fini », Ann. Sci. École Norm. Sup. 29 (1996), p. 185-272.

[4] P. Berthelot \& A. Ogus - Notes on crystalline cohomology, Princeton University Press, 1978.

[5] R. Bezrukavnikov, I. Mirković \& D. Rumynin - «Localization of modules for a semisimple Lie algebra in prime characteristic », Ann. of Math. 167 (2008), p. 945-991.

[6] S. Bosch, W. LÜtkebohmert \& M. Raynaud - Néron models, Ergebnisse der Mathematik und ihrer Grenzgebiete (3), vol. 21, Springer, 1990.

[7] N. Bourbaki - Éléments de mathématique, Chapitres 4, 5 et 6, Masson, 1981.

[8] J.-L. Brylinski \& M. Kashiwara - «Démonstration de la conjecture de Kazhdan-Lusztig sur les modules de Verma », C. R. Acad. Sci. Paris Sér. $A-B 291$ (1980), p. A373-A376. 
[9] M. Demazure - «Sous-groupes paraboliques des groupes réductifs », in Schémas en Groupes (Sém. Géométrie Algébrique, Inst. Hautes Études Sci., 1963/64), Fasc. 7, Exposé 26, Inst. Hautes Études Sci., 1966, p. 91.

[10] M. Demazure \& P. Gabriel - Groupes algébriques. Tome I : Géométrie algébrique, généralités, groupes commutatifs, Masson \& Cie, Éditeur, Paris, 1970.

[11] A. Grothendieck \& J. Dieudonné - «Éléments de Géométrie Algébrique, $1^{\text {re }}$ partie », Publ. Math. IHÉS, vol. 11, 1961.

[12] _ , «Éléments de Géométrie Algébrique, $4^{\mathrm{e}}$ partie », Publ. Math. IHÉS, vol. 32, 1967.

[13] B. HaAsterT - « Über Differentialoperatoren und D-Moduln in positiver Charakteristik », Manuscripta Math. 58 (1987), p. 385-415.

[14] C. Huyghe - « $\mathscr{D}^{\dagger}$-affinité de l'espace projectif», Compositio Math. 108 (1997), p. 277-318.

[15] J. C. Jantzen - Representations of algebraic groups, second éd., Mathematical Surveys and Monographs, vol. 107, Amer. Math. Soc., 2003.

[16] M. Kashiwara - «Representation theory and $D$-modules on flag varieties », Astérisque 173-174 (1989), p. 55-109.

[17] M. Kashiwara \& N. LAuritzen - « Local cohomology and $\mathscr{D}$-affinity in positive characteristic », C. R. Math. Acad. Sci. Paris 335 (2002), p. 993996.

[18] D. Kazhdan \& G. LuszTig - «Representations of Coxeter groups and Hecke algebras », Invent. Math. 53 (1979), p. 165-184.

[19] D. Mumford, J. Fogarty \& F. Kirwan - Geometric invariant theory, third éd., Ergebnisse der Mathematik und ihrer Grenzgebiete (2), vol. 34, Springer, 1994. 